\title{
Listening to ultrasound from plants reveals xylem vessel anatomy. Ultrasound characterization of plant vessels.
}

Satadal Dutta ( $\sim$ s.dutta-1@tudelft.nl )

TU Delft https://orcid.org/0000-0002-5817-5503

Elias Kaiser

Wageningen University and Research

Priscila Malcolm Matamoros

Wageningen University and Research

Peter Steeneken

Delft University of Technology https://orcid.org/0000-0002-5764-1218

Gerard Verbiest ( $\nabla$ g.j.verbiest@tudelft.nl )

Delft University of Technology

Article

Keywords: xylem vessel anatomy, ultrasound, non-invasive characterization

Posted Date: June 3rd, 2021

DOI: https://doi.org/10.21203/rs.3.rs-452046/v2

License: (9) This work is licensed under a Creative Commons Attribution 4.0 International License. Read Full License 


\section{Front Matter}

Title

- Listening to ultrasound from plants reveals xylem vessel anatomy.

- Ultrasound characterization of plant vessels.

\section{Authors}

Satadal Dutta, ${ }^{1 *}$ Elias Kaiser, ${ }^{2}$ Priscila Malcolm Matamoros, ${ }^{2}$ Peter G. Steeneken, ${ }^{1}$ Gerard J. Verbiest ${ }^{*}$

\section{Affiliations}

- $\quad{ }^{1}$ Department of Precision and Microsystems Engineering, Faculty of 3ME, TU Delft, Mekelweg 2, 2628CD Delft, the Netherlands.

- ${ }^{2}$ Horticulture and Product Physiology, Department of Plant Sciences, Wageningen University and Research, Droevendaalsesteeg 1, 6708PB Wageningen, the Netherlands.

- $\quad$ *Corresponding authors email: $\underline{\text { s.dutta-1@tudelft.nl, }}$ g.j.verbiest@tudelft.nl

\section{Abstract}

Although it is well known that plants emit ultrasound bursts under drought stress, the exact origin of the acoustic waveform of these pulses has remained elusive. Here we present evidence for a correlation between the ultrasound spectrum of these pulses and the dimensions of the plant's xylem vessels. Using a model that relates the vibrational excitations of the vessels to their geometric and viscoelastic properties, we develop a methodology to extract the internal xylem vessel dimensions from recorded ultrasound waveforms. We apply the method to ultrasound pulses from drying shoots of three vascular dicot plant species, and validate it by comparison with destructive measurements via microscopy. Our method demonstrates the potential for continuous monitoring of the vascular anatomy of plants. The ultrasonic, non-invasive characterization of internal vessel dimensions can lead to breakthroughs in speed and accuracy in plant phenotyping and disease detection in agriculture.

\section{Teaser}

Bursts of sound produced by plants convey the dimensions and elasticity of their watercarrying vessels.

\section{MAIN TEXT (max. 15,000 words)}

\section{Introduction}

The generation of sound waves by plants has received considerable attention during the last decades $(1,2)$. Several studies have investigated the mechanism of sound generation, and 
found that it is strongly related to plant hydraulics, which is linked to the mechanism of water transport in plants (3). This is vital to our understanding of plant function and stress resilience $(4,5)$. In vascular plants, the xylem is responsible for water and nutrient transport from the roots to the leaves (3). Evaporation of water from leaves (transpiration) results in a tensile force on the water-column, which, combined with the strong cohesion of water molecules, results in ascent of water from the roots to the leaves (6). During drought or strong transpiration rate, the tension in the water column can increase rapidly. Beyond a critical tension, the stress is released by the formation of vapor or gas-bubbles $(6-8)$ in the xylem. This bubble formation results in a sudden release of the elastic energy stored in the water column, a fraction of which is converted to a sound pulse (9). The rate at which such pulses are emitted has been used as a marker of a plant's response and vulnerability to drought-stress (10-13). The time- and frequency-domain features of these ultrasound pulses, measured directly from plant shoots, were shown in a recent study (14). Yet, the physical origin and relevance of the observed waveforms in these acoustic pulses (15-19) has remained elusive. Here, we present evidence that the waveforms are closely linked to the dimensions and mechanical properties of xylem vessels.

The xylem is a major player in determining a plant's response to biotic and abiotic stresses, e.g. availability of water, and pathogen infection. Monitoring xylem vessel traits is, thus beneficial for plant phenotyping. Current knowledge of the xylem vessel dimensions has mainly been dependent on microscopy techniques, and is important for the vibrational model that we will introduce later. Xylem vessels resemble cylindrical tubes with fused ends (5). These tubes consist of several xylem vessel elements that are separated by perforation plates. Diameters of these vessels range from $\sim 1 \mu \mathrm{m}$ in small herbs to $\sim 100 \mu \mathrm{m}$ in woody trees, and their lengths range from $\sim 100 \mu \mathrm{m}$ to $\sim 10 \mathrm{~cm}(20-22)$, across the plant kingdom. The viscoelastic walls of xylem cells are composed of an interwoven matrix of cellulose, hemicellulose, pectin and lignin fibres, which can have a wide range of elastic moduli depending on their relative composition (23) and the water content (24-26). The elasticity of macroscopic segments of plant stems can be measured via various mechanical loading techniques $(26,27)$, which are invasive. Existing techniques to measure xylem dimensions, such as paint-injection, X-ray micro-computed tomography (CT), optical microscopy and scanning electron microscopy (28-31), are also destructive and time-consuming.

Based on this knowledge of xylem vessels, we present a physical model that links their dimensions and (visco-)elasticity, to measured ultrasound pulses. Using Hydrangea quercifolia as a test species, we compare information about the radius, length, and viscoelasticity of xylem vessels obtained by analyzing ultrasound pulses, to that gained by independent destructive techniques. In addition, we further elucidate the correlation between viscous damping in the ultrasound pulses and the xylem vessel radius distribution by experiments on two additional species namely Hydrangea macrophylla, and Solanum lycopersicum. Lastly, via pulsed ultrasound spectroscopy with an external sound source, we show that acoustic resonances in the vascular tissue can be artificially excited, the characteristics of which agree with those excited naturally during drought-stress. As an analogy, our study suggests that xylem vessels serve as flutes inside a plant to communicate their physical state via the pitch and duration of their own 'music'. 


\section{Results}

Our study consists of two parts: (i) recording and analyzing ultrasound pulses from drying plant shoots, and (ii) validating the analytical results with destructive characterization on stem segments. The results section is organized as following: firstly, we analyze the time-domain features of the recorded ultrasound pulses. Secondly, we develop an analytical model to extract xylem vessel radius from the settling time of these pulses. These are validated by optical and scanning electron microscopy. Thirdly, we study the resonance frequencies present in the pulses. By combining this information with an independently measured Young's modulus of elasticity of the stem, we extract the length of xylem vessel elements; these lengths are validated via scanning electron microscopy. Fourthly, we merge our findings to create a map of acoustic lengths and radii of xylem vessels in a given species and also predict a theoretical relationship between length and radius in the context of viscous fluid flow and mechanical strength of cylindrical vessels. Lastly, we support our hypotheses by comparing the acoustic response of a plant stem under drought-stress to that when excited artificially with an external sound source.

\section{Recording ultrasound waveforms}

We first examine the waveforms of ultrasound pulses emitted by the drying plant shoots. A total of three specimens, samples A, B, and C, are taken from three H. quercifolia plants (see Materials and Methods). Ultrasound pulses are recorded with a broadband ultrasound microphone placed along the axial and radial direction of the stem, as shown in Fig. 1A. While the axial recording generally helps in detecting louder sound bursts (larger amplitudes), the radial recording is relevant from the viewpoint of application in non-invasive detection. The microphone records the time series of the ultrasound emissions starting $\sim 5$ minutes into the drying process (see Fig. 1B; Methods), where time $t=0 \mathrm{~s}$ corresponds to the start of the recording. The pulses occur sporadically and with varying amplitudes. We observe that the time-domain waveforms of these pulses resemble damped oscillations, both when recorded along the axial and in the radial directions (Figs. 1C and 1D). The pulse amplitude in timedomain decays exponentially with a 1/e time constant $\tau_{\mathrm{s}}$ : the settling time (Methods). For stem sample A, we obtained $\tau_{\mathrm{s}}=28.8 \pm 6.4 \mu$ s (mean \pm s.d.), for the pulses recorded in the axial direction. The corresponding value of $\tau_{\mathrm{s}}$ for the radially recorded pulses is $41.7 \pm 12.4 \mu \mathrm{s}$, which is statistically similar. The $\tau_{\mathrm{s}}$ for the many individually measured axial and radial sound pulses of all the three stem samples A, B, and C are shown in Figs. S1 - S3, respectively. The determined settling times of samples B and $\mathrm{C}$ agrees with those of sample A. All pulses die out within $\sim 0.3 \mathrm{~ms}$, in agreement with reported work (9). Based on this observation, we hypothesize that the damped oscillations are generated by resonant vibrations within the xylem vessels. In the following paragraphs, we develop a micromechanical model of the xylem vessel element based on acoustics in a pipe. In order to validate the model, we extract the settling times and characteristic frequencies of ultrasound pulse waveforms. These are subsequently interpreted to estimate xylem vessel dimensions and elasticity (see Fig. 1E).

\section{Pulse settling time and Xylem vessel radius}

In order to explain the origin of the observed ultrasound waveforms and to use them to extract information about the plant's microstructure, we develop a model relating the micromechanics of the xylem to the waveform of the generated ultrasound. We hypothesize that the damped oscillations are identical to those of an organ pipe filled with water (32). The 
bubble formation excites axial standing waves in the sap (water), whose resonance frequencies depend on the longitudinal speed of sound in the pipe $v_{\text {eff }}$, and the xylem vessel element length $L$ (Methods). We model the xylem vessel as a resonant cylindrical pipe containing a series network of vessel elements of length $L$, which are bounded by scalariform perforation plates $(3,28)$ (see Fig. 2A). The perforation plates serve as non-ideal (leaky) reflecting surfaces at the termination of a vessel element for the pressure waves. The sound waves propagate along the length of the xylem vessel, and are likely to dominate the recorded ultrasound. These waves undergo damping, primarily due to the dynamic viscosity of sap (water) $\eta_{1}$ in the xylem, which dominates the settling time $\tau_{\mathrm{s}}$. The resonating element is described using a linear second order resonator model consisting of lumped acoustic inductance, capacitance and resistance (Methods). Using this acoustic model, we express the effective xylem radius in terms of the sap density $\rho_{1}$ and measured settling time (see equation (8) in Materials and Methods). In this model, $R$ is calculated independently of length $L$, from the settling time of the measured time-domain waveform.

In order to validate our model, we obtain micrographs of the transverse cross-sections of the stem samples via optical microscopy (see Fig. 2B) and scanning electron cryo-microscopy (Fig. 2C). Figures 2B, and S4A-B show the optical micrographs of latex-paint stained stem samples $\mathrm{A}, \mathrm{B}$, and $\mathrm{C}$, respectively. Next, the histograms of xylem radii $R$ are extracted using the acoustic model from the axially recorded ultrasound pulses, and also from the optical micrographs. As shown in Fig. 2D, the mean ( \pm s.d.) acoustic $R$ for sample A is found to be $9.93 \pm 1.6 \mu \mathrm{m}$. The observed vessel radius $R$ via optical microscopy is $11.9 \pm 2.6 \mu \mathrm{m}$ (Fig. $2 \mathrm{D}$ and Table 1). Similar data are obtained for stem samples B and C (Figs. S4C-D). Thus, the calculated $R$, using the ultrasound analysis, agrees with that observed by optical and scanning electron microscopy.

We further validate our method using other plant species, namely $H$. macrophylla and $S$. lycopersicum (Figs. 2E - 2J). The mean $\tau_{\mathrm{s}}$ for H. Macrophylla and S. lycopersicum are $26.4 \pm 7.0 \mu \mathrm{s}$ and $116 \pm 85.0 \mu \mathrm{s}$, respectively. Histograms and mean $R$ derived from direct microscopy (see Figs. 2G, 2H), are in good agreement (see Figs. 2I, 2J). The relatively larger $\tau_{\mathrm{s}}$ for $S$. lycopersicum is in agreement with its wider mean vessel radius $(20.4 \pm 7.1 \mu \mathrm{m})$, compared to that of $H$. macrophylla $(10.9 \pm 2.4 \mu \mathrm{m})$. The corresponding vessel radii, obtained with the acoustic model, are $9.6 \pm 1.2 \mu \mathrm{m}$ and $20.5 \pm 8.6 \mu \mathrm{m}$ for the two species, respectively. The ultrasound methodology is thus validated for multiple plant species, showing the link between the vessel radii, and the settling time of the ultrasound pulses.

\section{Ultrasound frequencies, Xylem vessel (element) length and Young's modulus}

To estimate the length $L$ of the xylem vessel element, we analyse the frequencies in the ultrasound pulses. The resonance frequencies $f_{\mathrm{L}}$ are integer multiples of the ratio $v_{\mathrm{eff}} / L$ (see Materials and Methods). We observe that the Fourier spectra of representative ultrasound pulses (recorded axially) exhibit characteristic peak frequencies (Fig. 3A). The peak frequency with the largest amplitude, $f_{\mathrm{p} \text { (axial) }}$ for sample $\mathrm{A}$ is $34 \pm 5 \mathrm{kHz}$. In addition, peaks close to integral multiples of $f_{\mathrm{p} \text { (axial) }}$ are observed (Table S1). Analysis of pulses from samples $\mathrm{B}$ and $\mathrm{C}$ shows similar trends (Fig. S5A-B). Similar data are observed in the pulses recorded in the radial direction of the stem samples (Fig. 3B, Fig. S5B-C, and Table S2). 
expressed in terms of $f \mathrm{~L}$ in line with our acoustic pipe model (see equation (9) in Materials and Methods). In this model, the resonance frequency additionally depends on the mode order $\mathrm{m}$, the speed of sound in bulk water $v_{\mathrm{l}}$, the vessel wall thickness $h$ and the Young's modulus of elasticity $E$. The Young's modulus quantifies the stiffness of a solid against mechanical stress. It is defined as the amount of stress along a given direction, required to produce unit relative change in its dimension.

To extract $L$ from the resonance frequencies, we need to obtain $h$ and $E$ (see Materials and Methods). We observed $h$ to be $\sim 1 \mu$ m via scanning electron cryo-microscopy (see Fig. 2C). We determine $E$ of stem segments cut from the same plant, and from shoots similar in age and size. For this, we measure the stress-strain curves via uniaxial tensile loading (Fig. 3C). The mean mass density per stem segment is also estimated from the measured weights and dimensions. The linear slope of the stress-strain curve (Fig. 3C) at small values of strain $(\approx$ $10^{-4}$ ) yields the value of $E$, which is extracted to be $0.2 \pm 0.1 \mathrm{GPa}$ for fresh (hydrated) stem samples (Fig. 3D). For dry stem samples, $E>0.6 \mathrm{GPa}$ are obtained. We observe an overall decline in $E$ with increasing mass density. This indicates that the water-content dominates the variations in $E$. This agrees with an earlier empirical model (24), where the dependence of $E$ on the relative water content in the xylem is taken into account.

We calculate $L$ using $h \approx 1 \mu \mathrm{m}$ and $E=0.2 \pm 0.1 \mathrm{GPa}$ in equation (9) (see Materials and Methods). The histogram of $L$ is extracted from the axially recorded ultrasound pulses for stem samples. For sample A, $L$ is obtained to be $0.99 \pm 0.08 \mathrm{~mm}$ under a unimodal Gaussian fit (see Fig. 3E). Similar values are obtained for samples B and C (Fig. S5E-F). This highlights the reproducibility of our method and the similarity of the recorded ultrasound pulses in the axial direction.

Next, we validate the assumption that $L$ represents the actual length of xylem vessel element. We extract the mean xylem vessel length (a vessel contains several vessel elements) using latex paint staining (30), by counting the number of stained vessels on transverse crosssections of the stem (see Materials and Methods). These counts decrease exponentially with the distance (33) from the lower end of the stem at which the paint was taken up (Fig. S6). The mean xylem vessel lengths are found to be in the range $\sim 12-17 \mathrm{~mm}$ for the three stem samples. The xylem vessel length is thus much larger than the $L$ extracted from the ultrasound pulses $(\sim 1 \mathrm{~mm}$, Fig. 3E). This is because the Latex paint molecules cannot penetrate the fused ends, but can pass through the perforation plates between adjacent vessel elements (33). Therefore, we observe individual vessel elements in longitudinal sections of stem samples using scanning electron cryo-microscopy (Fig. 3F). The observed length ranges from 0.5 to $0.9 \mathrm{~mm}$ for individual xylem vessel elements (Fig. 3G, Table 1). Thus $L$, as obtained from our acoustic model, is a good estimate of the length of individual vessel elements.

\section{Relationship between $L$ and $R$}

Our method of analysing ultrasound emissions enables us to generate a set of length versus radius data for xylem vessel elements within a given stem segment. We observe that in a single plant (H. quercifolia), $L$ scales as $R^{0.74}$ (see Fig. 4). Basic fluid and structural mechanics helps us in predicting an upper bound on $L-R$ dependency. In plants of height within $\sim 1 \mathrm{~m}$, transpiration pull is the governing force of ascent of water through xylem vessels, which creates a gradient in the hydrostatic pressure along the vascular column. With a constant volume flow rate of water through the series-connected vessel elements (continuity), 
the pressure-drop along a length $L$ can be obtained from the Darcy-Weisbach equation (34) (see Materials and Methods). Furthermore, a vessel element can withstand a maximum pressure drop to avoid rupture (35). This critical pressure is also a function of both $L$ and $R$ (see Materials and Methods). Combining the two dependencies, we derive that $L_{\text {crit }} \propto R^{1.25}$, where Young's modulus and wall thickness are assumed to be constants. This reasoning gives us an upper-bound on the scaling exponent from a purely mechanical viewpoint.

\section{Ultrasound pulsed transmission spectroscopy of xylem vessels}

Finally, we further elucidate the link between acoustic resonances in xylem vessels to the drought-induced ultrasound pulses. For this, we artificially excite resonances in the vascular tissue of a stem segment of $H$. macrophylla. A piezo-transducer is used, which transmits an acoustic pulse when excited electrically (see Materials and Methods). This enables us to excite our target at a range of sound frequencies simultaneously. The pulse is applied such that it propagates through the stem segment along either the axial or the radial direction, as shown in Fig. 5A. The transmitted sound pulse is subsequently detected by the broad-band microphone. Figures 5B and 5C show the time-domain and frequency-domain waveform of the ultrasound pulse detected axially, while Figs. 5D and 5E show the same for the pulse detected radially. The ultrasound pulse exhibits an envelope settling time of $36.3 \mu \mathrm{s}$, which is in close agreement with that obtained from the drought-induced ultrasound pulses $(26.4 \pm 7.0$ $\mu \mathrm{s})$. In both axial and radial directions, characteristic frequencies are observed in the Fourier spectra, which match those observed in drought-induced pulses. This could enable the use of acoustic excitation as a technique for non-invasive monitoring of vascular geometry and moisture-dependent elasticity.

\section{Discussion}

Our results have shown how ultrasound emissions from drought-stressed plant stems can be used to extract and monitor the geometry and viscoelasticity of xylem vessels. In this section, we first interpret our results and discuss the applicability of our method to monitor the vascular physiology of plants. We end the section by commenting on its potential in noninvasive plant health monitoring.

\section{Xylem vessel radius}

We have shown that by modelling the xylem vessel as cylindrical acoustic resonator, the radius $R$ can be extracted from the settling time of the ultrasound pulse, resulting in comparable values as those obtained from common microscopy techniques. Using Hydrangea and Solanum as example plant species with relatively narrow and wide vessel radii respectively, we validate the dependency of $\tau_{\mathrm{s}}$ on $R$. Optically determined xylem vessel radii are slightly bigger $(\sim 2 \mu \mathrm{m})$ than the acoustically determined radii (Figs. 2D, 2I, 2J, Fig. S4). We attribute this to the assumption of a constant dynamic viscosity of xylem sap $\eta$. In practice, $\eta_{1}$ depends on ambient temperature, and concentration of dissolved nutrients (36). Moreover, water close to the sap-wall interface is held with adhesive forces, and thus has a slightly higher dynamic viscosity (37). As a corollary to our analysis, if the distribution of $R$ is known directly from optical microscopy, one can evaluate the effective kinematic viscosity $(\eta 1$ $\left./ \rho_{1}\right)$ of the xylem sap. Note that the solid walls of the xylem vessels also possess shear or extensional viscosity (38). This means that elastic forces arise in them as a response to 
elongation, compression or shear stresses. Shear viscosity is a property of solids to resist a change in deformation (shear rate). This additional viscosity likely sets an upper bound on $\tau_{\mathrm{s}}$ and $R$, beyond which the agreement between optical and acoustic radii likely deteriorates.

\section{Xylem vessel element length $(L)$ and Young's modulus $(E)$}

The xylem vessel element length $L$, extracted from the ultrasound pulses (Fig. 3E) consistently exceeds the physical length (via SEM; Fig. 3G) by $\sim 0.3 \mathrm{~mm}(\sim 30 \%)$. We attribute this to two factors. Firstly, the perforation plates serve as non-rigid and leaky boundaries (not accounted for in the model), due to which the standing waves penetrate beyond the physical length of a single vessel element. Secondly, the uniaxial tensile loading measurements that we performed (Fig. 3C, 3D) on stems provide an overestimation of the xylem Young's modulus. This is due to the presence of stiffer Sclerenchyma and Collenchyma tissue (39), with Young's moduli exceeding $~ 1 \mathrm{GPa}(40)$, close to the circumference of the stem. Hence, as a corollary to our analysis, instead of fixing the Young's modulus, one can alternatively fix the xylem vessel element length via microscopy. Xylem cells differentiate very early during the growth of a plant (41), subsequently growing to their maximum lengths before maturing (dying) (42) to become hydraulically active vessel elements. Thus, once the vessel element length is determined via microscopy techniques for a given plant, the Young's modulus can then be continuously and non-invasively monitored to diagnose variations in water-content (43), ageing, or even pathogen-induced occlusions within the xylem $(44,45)$.

\section{Application to intact plants}

Plants vary in their drought-resistance. It may take several days for the water potential in the leaves to fall below the reported threshold (10) for cavitation based ultrasound emissions of at least one per minute. Therefore, we detached plant shoots to induce accelerated droughtstress. This enabled us to record a large set of ultrasound pulses in a relatively short time. We measured similar waveforms in both axial and radial directions of the stem. The latter direction avoids physical incision of the stem and is, therefore, preferred for non-invasive measurements on intact plants. Ultrasound does not propagate far and events occurring within a maximum distance of $20-30 \mathrm{~mm}$ are likely to be useful (46). This distance depends on the species, and the level of hydration in the stem, and thus adjusting the proximity of the microphone to the stem may be necessary during growth or movements of the plant. For large trees/shrubs, the radius and lengths of the xylem vessels exceed $\sim 100 \mu \mathrm{m}$, and $\sim 10 \mathrm{~cm}$. This would require shifting the sensitive frequency band of the microphone down to the audible range $(100 \mathrm{~Hz}-10 \mathrm{kHz})$.

Impact and scope. The current state-of-the-art for determining xylem vessel properties is largely centred on cutting stems and examining them under a microscope, which comes with obvious drawbacks to speed and scalability. Using methods like latex paint staining and scanning electron microscopy to monitor xylem vessels is time-consuming, and is of limited applicability in the field. Recently, X-ray micro-tomography was recommended (47) to monitor xylem embolisms and hydraulic vulnerability. However, to date, this method is expensive and not suitable for field applications. Ultrasound monitoring far surpasses these techniques, and has the potential to monitor xylem vessels non-destructively and continuously with a relatively inexpensive apparatus. The presented methodology establishes a link between geometrical and mechanical properties of xylem vessels and the recorded ultrasound 
emissions of plants. In particular, we showed, for the first time, the potential of ultrasound monitoring in the rapid determination of radius $(R)$ and length $(L)$ of xylem vessel elements in a single plant (Fig. 4). This opens the route to new studies about any existing physical relationship between $L$ and $R$ in a single plant. Note that although observed values of $L$ and $R$ were reported in literature $(28,47,48)$, those were obtained only across different species by either destructive microscopy or X-ray micro-tomography.

We foresee applications of our method to a multitude of plant species with varying vessel dimensions and viscoelasticity. This can enable in-vivo studies to mechanical resonances of a plants' vascular tissue via external acoustic transducers. In turn, this provides a non-invasive method for rapid phenotyping. Crops could be selected for breeding based on their xylem vessels and thus based on their response to drought and/or susceptibility to vascular wilt pathogens $(41,42,49,50)$. Drought-stress directly impacts the viscoelasticity of the vascular tissue, which can be monitored with ultrasound. Correlation between vessel radius and drought-stress have been reported in poplar (51) and apple trees (52). Pathogens within the xylem vessels have a parasitic effect on the sugar/nutrient concentration in the sap, which can in turn change the kinematic viscosity of the xylem sap.

Lastly, from the viewpoint of a complete sensor system, the presented methodology only uses Fourier transforms and envelope detection. These are standard signal processing functions, which can be implemented in commercial integrated chip technology. This will help with future development of low-cost and compact tools for monitoring plant stress. This will in turn boost climate-smart agriculture, and indoor farming by providing farmers with new tools for optimal irrigation strategies and early disease-detection. The presented methodology provides a new outlook on plants "talking" during drought-stress, and presents ultrasound sensing as an inexpensive technique for rapid, non-invasive and in-vivo characterization of plant vasculature.

\section{Materials and Methods}

\section{Plant material}

Three potted plants of Hydrangea quercifolia were obtained from a commercial garden centre and moved to the laboratory within 1 hour. One shoot sample per plant was cut, keeping the leaves intact, and immediately placed in tap water (Fig. S7) to prevent embolism in the xylem vessels at the cut-end. From each shoot sample, a 60-70 mm long and trimmed (i.e., without leaves and petioles) stem segment was cut under water to prevent air entry and blockage. The segments were roughly cylindrical, with a cross-section diameter of $\sim 5-6 \mathrm{~mm}$, and were used for vessel staining and optical microscopy. The rest of the sample was left intact to measure ultrasound emissions. Additionally, one plant each of Hydrangea macrophylla and Solanum lycopersicum, was also obtained for optical microscopy and ultrasound recording.

\section{Recording ultrasound pulses and signal processing}

The shoot samples were taken out of water, dried using tissue paper, and left on the bench for air-drying, resulting in accelerated drought stress. A M500-USB ultrasound microphone, with a reliable detection window between $10 \mathrm{kHz}$ and $150 \mathrm{kHz}$, from Pettersson Elektronik AB 
(Uppsala, Sweden) was placed first in the axial ( $2 \mathrm{~mm}$ from the cut-face of stem normal to the cross-section) and then in the radial (on the cylindrical surface of the stem) directions (Fig. 1A) to record the ultrasound bursts at a sampling rate of $500 \mathrm{kHz}$ in continuous time windows of 120 seconds. The sensor consists of a piezoelectric material which produces an electrical voltage proportional to the pressure of the incident sound wave. From the timedomain waveforms, the pulse envelope was obtained with the built-in "envelope ()" function in MATLAB, which returns the upper and lower envelopes of the input sequence, as the magnitude of its analytic signal. The analytic signal of the input sequence was found using the Hilbert transform. The peak of the envelope curve was determined and the decreasing part of the envelope curve was stored, which was subsequently fitted with the exponential function $\exp \left(-t / \tau_{\mathrm{s}}\right)$ using the Least-Squares method. This yielded the settling time $\tau_{\mathrm{s}}$. The frequency spectra of the measured signals were obtained via a 250-point Discrete Fourier Transform, spanning a time frame of $1.5 \mathrm{~ms}$. Due to the low intensity of the emitted sound, the spectra are shown until $150 \mathrm{kHz}$ beyond which the signal merges with the noise floor of the sensor ($80 \mathrm{~dB}$ ). The raw data was then post-processed and analysed in MATLAB R2018b (MathWorks, Massachusetts, USA).

\section{Analytical model for longitudinal vibrations}

We modelled the xylem vessel as a cylindrical pipe of radius $R$, and effective length $L$ sustaining longitudinal standing waves $(53,54)$ in the water of density $\rho$ 1 whose resonance frequencies depend on the mode order $\mathrm{m}$, and the longitudinal speed of sound in the pipe $v_{\text {eff. }}$ The resonant frequency of the $\mathrm{m}^{\text {th }}$ order $(\mathrm{m}=1,2, \ldots)$ is given by

$$
f_{\mathrm{m}}=\left(\frac{\mathrm{m}}{2}\right) \frac{v_{\mathrm{l}}}{L}
$$

where $v_{\mathrm{l}}$ is the speed of sound in the liquid $\left(\sim 1482 \mathrm{~m} / \mathrm{s}\right.$ in bulk water at $\left.20{ }^{\circ} \mathrm{C}\right)$. We denote the fundamental resonance frequency $(\mathrm{m}=1)$ as $\mathrm{f}$ in the rest of this section. In practice, equation (1) cannot be applied directly because in a real pipe with an elastic wall, sound propagates at a slower speed than that in the bulk liquid. If the walls of the pipe have a non-zero acoustic thickness $h$ and finite Young's modulus $E$, then the effective speed of sound is given by $(55,56)$ :

\section{density of water. Thus, $v_{1}$ is replaced by $v_{\text {eff }}$ in equation (1).}

where $\beta_{\text {xylem }}$ is known as the cross-sectional compressibility, and $\rho 1=996 \mathrm{~kg} \cdot \mathrm{m}^{-3}$ is the mass

These sound waves (expected to be dominant in the axially recorded ultrasound) undergo damping primarily due to the dynamic viscosity of water (57) $\eta_{1}$ in the xylem. The resulting time-domain response of the resonating pipe can be described using a lumped circuit model consisting of acoustic inductance $\left(L_{\mathrm{a}}\right)$, capacitance $\left(C_{\mathrm{a}}\right)$ and resistance $\left(R_{\mathrm{a}}\right)$, analogous to an 
electrical L-C-R circuit, where voltage and current are replaced by pressure and flow rate respectively. $L_{\mathrm{a}}$ is a consequence of the kinetic energy in the water, while $C_{\mathrm{a}}$ arises due to the compressibility of water. $R_{\text {a }}$ leads to energy dissipation and can be obtained from Poiseuille's law for capillary flow $(38,53)$. The three lumped parameters can be expressed as:

$$
L_{\mathrm{a}}=\frac{L \cdot \rho_{\mathrm{l}}}{\pi \cdot R^{2}}, \quad C_{\mathrm{a}}=\frac{L \cdot \pi \cdot R^{2}}{\rho_{\mathrm{l}} \cdot v_{\mathrm{l}}^{2}}, \quad R_{\mathrm{a}}=\frac{8 \cdot \eta_{\mathrm{l}} \cdot L}{\pi \cdot R^{4}}
$$

where $\eta_{1}=8.9 \times 10^{-4} \mathrm{~Pa}$.s is the dynamic viscosity of water. By describing the circuit as a linear $2^{\text {nd }}$ order differential equation, we obtained the damping ratio $\zeta$, envelope settling time $\tau_{\mathrm{s}}$ (the time needed for the amplitude to decrease by a factor of ' $e$ '), and the driving frequency $f_{\mathrm{d}}$ as :

The lumped model is valid as long as the dimensions $L$ and $R$ are smaller than the acoustic wavelength $(\sim 1-10 \mathrm{~cm}$ in water $)$.

Noting that $f_{\mathrm{d}}$ is the same as the observed $f_{\mathrm{p}}$ (axial) in the ultrasound pulses, $\zeta$ is obtained by combining equations (5) and (6) as :

$$
\zeta=\frac{1}{\sqrt{1+\left(f_{\mathrm{p}(\text { axial })} \cdot \tau_{\mathrm{s}}\right)^{2}}}
$$

And the acoustic xylem radius was obtained by rearranging equation (5):

$$
R=\sqrt{\frac{4 \cdot \eta_{\mathrm{l}} \cdot \tau_{\mathrm{s}}}{\rho_{\mathrm{l}}}}
$$

Combining equations (1) and (2), the effective xylem length $L$ was obtained as: 


$$
\frac{1}{L^{2}}=\frac{4 f_{\mathrm{L}}^{2}}{\mathrm{~m}^{2} v_{\mathrm{eff}}^{2}}=\frac{4 f_{\mathrm{L}}^{2}}{\mathrm{~m}^{2}}\left[\frac{1}{v_{\mathrm{l}}^{2}}+\frac{2 \rho_{\mathrm{l}} R}{h} \cdot\left(\frac{1}{E}\right)\right]
$$

412

413

\section{Scanning electron (cryo-) microscopy}

Transverse sections from hydrangea stems were made using a razorblade. The cross-section was left on filter paper for 1-2 minutes to remove most of the adhering water. Thereafter, the section was fixed to a sample holder using Tissue-Tek. The sample was frozen by plunging the sample holder into liquid nitrogen. Subsequently the sample was transferred to a cryopreparation chamber (Leica Microsystems, Wetzlar, Germany) under vacuum where it was kept at $-90^{\circ} \mathrm{C}$ for 3 minutes to remove ice from the surface (freeze etching to remove water vapor contamination). While still under vacuum the sample was coated with $12 \mathrm{~nm}$ of tungsten and transferred using a VCT100 shuttle (Leica) to a field emission scanning electron microscope (Magellan 400 from FEI, Oregon, USA). The samples were analysed at $2 \mathrm{kV}, 13$ pA at $-120^{\circ} \mathrm{C}$.

Longitudinal sections were made by carefully cutting through the region that contains the xylem vessels. The rest of the sample preparation was identical.

\section{Uniaxial tensile loading for Young's modulus determination}

Multiple stem segments of lengths in the range of $4-7 \mathrm{~cm}$ were cut and mounted vertically between two clamps of a tensile testing machine (Z005; Zwick/Roell, Ulm, Germany; inset of Fig. 3C). The initial pre-strained length $\left(l_{0}\right)$ is equal to the vertical separation between the clamps and was kept as $20 \mathrm{~mm}$. The uniaxial stress was calculated as the tensile force applied by the equipment divided by the average cross-section area of the stem segment. The longitudinal strain was calculated as the change in stem length per unit initial length $\left(\Delta l / l_{0}\right)$. The Young's modulus $E$ was then extracted as the slope of the linear part of the stress-strain curve (Fig. $3 \mathrm{c}$ ) at small values of strain $\left(\approx 10^{-4}\right)$. The average mass density of each sample was also calculated from measured weight and volume just before tensile loading. The weights were measured with a Scaltec SBC 33 precision balance (Scaltec Instruments GmbH, Göttingen, Germany), while the dimensions were measured with a standard Vernier Calliper with a resolution of $0.1 \mathrm{~mm}$. Note that the measurement error for elastic moduli and mass density $(\sim 20 \%)$ is predominantly due to error propagation from length and diameter measurements.

\section{Vessel staining and optical microscopy}

An aqueous solution $1 \%(\mathrm{v} / \mathrm{v})$ suspension of red latex paint was left standing for at least 24 hours to allow large particles to settle at the bottom. The supernatant was subsequently transferred to a glass container and degassed. The stem segments were mounted vertically over the glass container, with one end immersed in the paint and the other end tightly inserted into a plastic tube connected to a suction pump (Fig. S7) which applied a pressure difference of 400 mbar. The stem-tube junction was taped and smeared with Vaseline to prevent air leakage. As the solution was sucked through the stem for 12 hours, the paint remained confined in one xylem vessel (macromolecules in the paint cannot move through the 
bordering pits of xylem vessels) while the clear water was conducted through the entire stem. Subsequently, the stem samples were sliced with a blade at intervals of $5 \mathrm{~mm}$. The number of painted vessels was then counted on each face of the cut slices from images (magnification of 200X) captured by a VHX digital microscope from Keyence.

An exponential relationship was observed (58) for the number of continuous xylem vessels at varying lengths of a stem segment. Typically, it is observed that longer vessels are also wider (59). The complex relationship between xylem radius and length in a plant is largely affected by a trade-off between hydraulic conductance (increases with increasing $R$ and decreasing $L$ ), and vulnerability to cavitation (60) (increases with increasing $R$ and $L$ ). The xylem vessel length has the following probability distribution function $(58,60)$ :

$$
P(x)=x \cdot \lambda_{\text {xylem }}^{2} \exp \left(-\lambda_{\text {xylem }} x\right)
$$

where the most probable vessel length is given by $\lambda^{-1}$ xylem, while the mean and standard deviation are respectively given by $2 \lambda^{-1}$ xylem, and $1.414 \lambda^{-1}$ xylem. Equation (10) is based on the assumption that xylem vessels have, for every additional unit length, a similar chance to terminate (58). Thus, we describe the length distribution of a vessel population. Starting from a chosen reference position $x=0$, the number of vessels $N$ with length $L \geq x$ is given by

$$
N=N_{0} \exp \left(-\lambda_{\text {xylem }} x\right)
$$

\section{Ultrasound pulsed transmission spectroscopy}

A stem segment $43 \mathrm{~mm}$ in length and $5 \mathrm{~mm}$ in diameter was obtained from a H. macrophylla plant. For detecting axially transmitted ultrasound pulse through the stem, a piezo-transducer (MA40S4S; Murata Manufacturing Co., Ltd., Kyoto, Japan) with a resonant frequency of 40 $\mathrm{kHz}$ was excited with a voltage step of $10 \mathrm{~V}$ (zero offset), and an on-time of $500 \mathrm{~ms}$. The transducer was placed perpendicular to one end of the stem segment. The M500-USB microphone was placed perpendicular to the other end of the stem segment. The frequency spectra of the measured signals were obtained via a 250-point Discrete Fourier Transform, spanning a time frame of $1.5 \mathrm{~ms}$. The raw data was then post-processed and analysed in MATLAB R2018b (MathWorks, Massachusetts, USA). For radial transmission, the same stem segment was mounted between the transducer and the microphone such that the longitudinal axis of the stem was perpendicular to the line of flight of the sound pulse (Fig. 4A). The transducer was excited with a voltage step of $5 \mathrm{~V}$ and an on-time of $500 \mathrm{~ms}$. The Fourier transform of the detected ultrasound pulse was performed over a time span of the first $100 \mu$ s (Fig. 4D), to observe the frequency components present in the pulse that propagated only through the stem.

\section{Darcy-Weisbach equation and critical pressure in xylem vessel}

The Darcy-Weisbach equation is an empirical relation that relates the pressure drop $p$ along a given length $L$ of a viscous and incompressible fluid flowing through a conduit of radius $R$ as: 


$$
p=\frac{8 \eta_{l} Q L}{\pi R^{4}}
$$

491

492 where $Q$ is the volumetric flow rate and $\eta_{1}$ is the dynamic viscosity of the fluid (water).

493

494

495

496

497

498

499

500

501

502

503

504

505

506

507

508

509

510

511

512

513

514

515

516

517

518

519

From the viewpoint of mechanical rupture/failure, a biomechanical model was reported (35) where a vessel element is treated as a cylindrical shell under hydrostatic pressure of length $L$, radius $R$, uniform wall thickness $t$, and isotropic homogeneous Young's modulus E. For cylinders with $L /(R . t)^{0.5}>4$ (applicable for xylem vessel elements), the critical hydrostatic pressure $p_{\text {crit }}$ can be found from Batdorf's approximate formula $(35,62)$ as:

$$
p_{\text {crit }}=\frac{0.92 t^{5 / 2} E}{L \cdot R^{3 / 2}}
$$

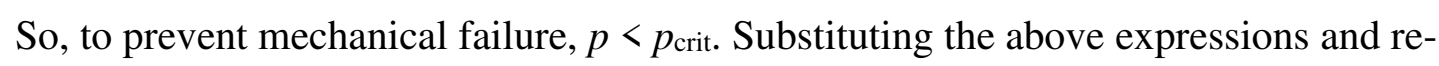
arranging the terms, we obtain:

$$
L<\left(\sqrt{\frac{0.92 \pi t^{5 / 2} E}{8 \eta_{l} Q}}\right) \cdot R^{5 / 4}
$$

\section{Supplementary Materials}

Supplementary material for this article is available.

\section{References}

1. M. Gagliano, Green symphonies: a call for studies on acoustic communication in plants. Behavioral Ecology 24(4), 789-796 (2012).

2. S.B. Kikuta, M.A. Lo Gullo, A. Nardini, H. Richter, S. Salleo, Ultrasound acoustic emissions from dehydrating leaves of deciduous and evergreen trees. Plant, Cell and Environment 20(11), 1381-1390 (1997).

3. M.D. Venturas, J.S. Sperry, U.G. Hacke, Plant xylem hydraulics: What we understand, current research, and future challenges. J. Integr. Plant Biol. 59(6), 356-389 (2017).

4. N.G. McDowell, T.J. Brodribb, A. Nardini, Hydraulics in the $21^{\text {st }}$ century. New Phytologist 224, 537-542 (2019).

5. B. Choat, T.J. Brodribb, C.R. Brodersen, R.A. Duursma, R. López, B.E. Medlyn, Triggers of tree mortality under drought. Nature 558, 531-539 (2018). 
6. U. Zimmermann, A. Haase, D. Langbein, F. Meinzer, Mechanisms of long-distance water transport in plants: a re-examination of some paradigms in the light of new evidence. Phil.Trans. R. Soc. Lond. B 341, 19-31 (1993).

7. F. Caupin, E. Herbert, Cavitation in water: a review. C. R. Physique 7, 1000-1017 (2006).

8. H.J. Schenk, K. Steppe, S. Jansen, Nanobubbles: a new paradigm for air-seeding in xylem. Trends in Plant Sci. 20(4), 199-205 (2015).

9. A. Ponomarenko, O. Vincent, A. Pietriga, H. Cochard, É. Badel, P. Marmottant, Ultrasonic emissions reveal individual cavitation bubbles in water-stressed wood. J. $R$. Soc. Interface 11, 20140480 (2014).

10. G.E. Jackson, J. Grace, Field measurements of xylem cavitation: are acoustic emissions useful? J. Exp. Botany 47(11), 1643-1650 (1996).

11. S. Rosner, Acoustic emission related to drought stress response of deciduous broadleaved woody species. J. Acoustic Emission 30, 11-20 (2012).

12. M. Nolf, B. Beikircher, S. Rosner, A. Nolf, S. Mayr, Xylem cavitation resistance can be estimated based on time-dependent rate of acoustic emissions. New Phytologist 208, 625-632 (2015).

13. P. Dostál, P. Sriwongras, V. Trojan, Detection of acoustic emission characteristics of plant according to water stress condition. Acta Univ. Agric. Silvic. Mendel. Brun. 64(5), 1465-1471 (2016).

14. D. Oletic, S. Rosner, M. Zovko, V. Bilas, Time-frequency features of grapevine's xylem acoustic emissions for detection of drought stress. Computers and Electronics in Agriculture 178, 105797 (2020).

15. K.T. Ritman, J.A. Milburn, Acoustic emissions from plants: ultrasonic and audible compared. J. Exp. Botany 39(9), 1237-1248 (1988).

16. R. Zweifel, F. Zeugin, Ultrasonic acoustic emissions in drought-stressed trees - more than signals from cavitation? New Phytologist 179, 1070-1079 (2008).

17. L.L. Vergeynst, M.G.R. Sause, N.J.F. De Baerdemaeker, L. De Roo, K. Steppe, Clustering reveals cavitation-related acoustic emission signals from dehydrating branches. Tree Physiology 36, 786-796 (2016).

18. L.L Vergeynst, M.G.R. Sause, M.A. Hamstad, K. Steppe, Deciphering acoustic emission signals in drought stressed branches: the missing link between source and sensor. Front. Plant Sci. 6:494 (2015).

19. I. Khait, et al., https://www.biorxiv.org/content/10.1101/507590v4.full (2019).

20. U.G. Hacke, R. Spicer, S.G. Schreiber, L. Plavcová, An ecophysiological and developmental perspective on variation of vessel diameter. Plant Cell and Environment 40, 831-845 (2017). 
21. M.S. Smith, J.D. Fridley, J. Yin, T.L. Bauerle, Contrasting xylem vessel constraints on hydraulic conductivity between native and non-native woody understory species. Front. Plant Sci. 4, 486 (2013).

22. A.L. Jacobsen, R.B. Pratt, M.F. Tobin, U.G. Hacke, F.W. Ewers, A global analysis of xylem vessel length in woody plants. Amer. J. Bot. 99(10), 1583-1591 (2012).

23. L.J. Gibson, The hierarchical structure and mechanics of plant materials. J. R. Soc. Interface 9, 2749-2766 (2012).

24. K.J. Niklas, Plant biomechanics: an engineering approach to plant form and function (The University of Chicago Press, 1992).

25. K. Schulgasser, A. Witztum, On the strength of herbaceous vascular plant stems. Annals of Botany 80, 35-44 (1997).

26. K.J. Niklas, F.C. Moon, Flexural stiffness and modulus of elasticity of flower stalks from Allium stalks from Allium sativum as measured by multiple resonance frequency spectra. Amer. J. Bot. 75(10), 1517-1525 (1988).

27. D.U. Shah, T.P.S. Reynolds, M.H. Ramage, The strength of plants: theory and experimental methods to measure the mechanical properties of stems. J. Exp. Botany 68(16), 4497-4516 (2017).

28. C. van der Schoot, A.J.E. van Bel, Architecture of the internodal xylem of tomato (Solanum lycopersicum) with reference to longitudinal and lateral transfer. Amer. J. Bot. 76(4), 487-503 (1989).

29. A.L. Jacobsen, F.D. Rodriguez-Zaccaro, T.F. Lee, J. Valdovinos, H.S. Toschi, J.A. Martinez, R.B. Pratt, Functional and ecological xylem anatomy: Grapevine xylem development, architecture, and function Ch. 5 (Springer International Publishing Switzerland, 2015).

30. R. Pen, J. Geng, J. Cai, M.T. Tyree, A comparison of two methods for measuring vessel length in woody plants. Plant, Cell, and Enviroment 38, 2519-2526 (2015).

31. C.R. Brodersen, T. Knipfer, A.J. McElrone, In-vivo visualization of the final stages of xylem vessel refilling in grapevine (Vitis vinifera) stems. New Phytologist 217, 117 126 (2018).

32. J. Angster, P. Rucz, A. Miklós, Acoustics of organ pipes and future trends in the research. Acoustics Today 13(1), 10-18 (2017).

33. F.W. Ewers, J.B. Fisher, Techniques for measuring vessel lengths and diameters in stems of woody plants. Amer. J. Bot. 76(5), 645-656 (1989).

34. H. Rouse, Elementary Mechanics of Fluids (New York: John Wiley \& Sons Inc., 1946).

35. G.N. Karam, Biomechanical model of the xylem vessel elements in vascular plants. Annals of Botany 95, 1179-1186 (2005). 
36. V.R.N. Telis, J. Telis-Romero, H.B. Mazzotti, A.L. Gabas, Viscosity of aqueous carbohydrate solutions at different temperatures and concentrations. International Journal of Food Properties 10(1), 185-195 (2007).

37. M.P. Goertz, J.E. Houston, X.-Y. Zhu, Hydrophilicity and the viscosity of interfacial water. Langmuir 23, 5491-5497 (2007).

38. S. Vogel, Life in moving fluids: The physical biology of flow (Princeton University Press, 1994).

39. O. Leroux, Collenchyma: a versatile mechanical tissue with dynamic cell walls. Annals of Botany 110, 1083 - 1098 (2012).

40. O. Treitel, Elasticity of plant tissues. Transactions of the Kansas Academy of Science 47(2), $219-239$ (1944).

41. K. Růžička, R. Ursache, J. Hejátko, Y. Helariutta, Xylem development-from the cradle to the grave. New Phytologist 207(3), 519 - 535 (2015).

42. A.L. Jacobsen, J. Valdovinos-Ayala, R.B. Pratt, Functional lifespans of xylem vessels: development, hydraulic function, and post-function of vessels in several species of woody plants. Amer. J. Bot. 105(2), 142 - 150 (2018).

43. G. von Arx, S.R. Archer, M.K. Hughes, Long-term functional plasticity in plant hydraulic architecture in response to supplemental moisture. Annals of Botany 109, 1091-1100 (2012).

44. K.A. Yadeta, B.P.H.J. Thomma, The xylem as battleground for plant hosts and vascular wilt pathogens. Front. Plant Sci. 4: 97, (2013).

45. J. Pouzoulet, E. Scudiero, M. Schiavon, P.E. Rolshausen, Xylem vessel diameter affects the compartmentalization of the vascular pathogen Phaeomoniella chlamydospora in grapevine. Front. Plant. Sci. 8: 1442, (2017).

46. M.T. Tyree, J.S. Sperry, Characterization and propagation of acoustic emission signals in woody plants: towards an improved acoustic emission counter. Plant, Cell, and Environment 12, 371-382 (1989).

47. H. Cochard, S. Delzon, E. Badel, X-ray microtomography (micro-CT): A reference technology for high-resolution quantification of xylem embolism in trees. Plant Cell Environ. 38, 201-206 (2015).

48. S. Helmling, A. Olbrich, I. Heinz, G. Koch, Atlas of vessel elements. International Association of Wood Anatomists J. 39(3), 249-352 (2018).

49. C.J. Blackman, T.J. Brodribb, G.J. Jordan, Leaf hydraulic vulnerability is related to conduit dimensions and drought resistance across a diverse range of woody angiosperms. New Phytologist 188, 1113-1123 (2010).

50. M. Haworth, M. Centritto, A. Giovannelli, G. Marino, N. Proietti, D. Capitani, A. De Carlo, F. Loreto, Xylem morphology determines the drought response of two Arundo donax ecotypes from contrasting habitats. GCB Bioenergy 9, 119-131 (2017). 
657

\section{8}

659

660

661

662

663

664

665

666

667

668

51. M. Arend, J. Fromm, Seasonal change in the drought response of wood cell development in poplar. Tree physiol. 27, 985 - 992 (2007).

52. T.L. Bauerle, M. Centinari, W.L. Bauerle, Shifts in xylem vessel diameter and embolisms in grafted apple trees of differing rootstock growth potential in response to drought. Planta 234, 1045-1054 (2011).

53. L.E. Kinsler, A.R. Frey, Fundamentals of Acoustics, $2^{\text {nd }}$ ed. (New York: John Wiley \& Sons, 1962).

54. A. Wood, Acoustics, $2^{\text {nd }}$ ed. (New York: Dover, 1966).

55. A.S. Tijsseling, Exact solution of linear hyperbolic four-equation system in axial liquid-pipe vibration. J. Fluids and Structures 18(2), 179-196 (2003).

56. M.S. Ghidaoui, M. Zhao, D.A. McInnis, D.H. Axworthy, A review of water hammer theory and practice. Appl. Mech. Rev. 58(1), 49-76 (2005).

57. A.A. Aleksandrov, M.S. Trakhtengerts, Viscosity of water at temperatures of -20 to $150{ }^{\circ}$ C. J. Engineering Phys. 27, 1235-1239 (1974).

58. J. Nijsse, On the mechanism of xylem vessel length regulation. Plant Physiology 134(1), 32-34 (2004).

59. U.G. Hacke, J.S. Sperry, J.K. Wheeler, L. Castro, Scaling of angiosperm xylem structure with safety and efficiency. Tree Physiology 26, 689-701 (2006).

60. H. Ooeda, I. Terashima, H. Taneda, Intra-specific trends in lumen and wall resistivities of vessels within the stem xylem vary among three woody plants. Tree Physiology 38, 223-231 (2017).

61. J.P. Comstock, J.S. Sperry, Theoretical considerations of optimal conduit length for water transport in vascular plants. New Phytol. 148, 195-218 (2000).

62. L. Kollàr, E. Dulàcska, Buckling of shells for engineers (New York: John Wiley \& Sons Inc., 1984).

\section{Acknowledgments}

This work has been carried out under the "Plantenna" research programme, a collaboration among the members (technical universities) of the 4TU federation in the Netherlands. The authors would like to thank Lars Pettersson for technical support concerning the ultrasound sensor equipment, dr. ing. Marcel Giesbers for support with scanning electron microscopy, and Patrick van Holst for technical support in measuring the stress-strain curves of the stem samples.

Funding: Dutch 4TU Federation. 
677

678 679

680 681

682

683

684

685

686

687

688

689

690

691

692

693

694

695

696

697

698

699

\section{Author contributions:}

Conceptualization: SD, GJV

Methodology: SD, PMM, EK

Investigation: SD, PMM, GJV

Visualization: SD, GJV

Supervision: EK, GJV, PGS

Writing-original draft: SD

Writing_review \& editing: EK, GJV, PGS

Competing interests: The authors declare no competing interests.

Data and materials availability: The data that support the findings of this study are available from the corresponding author upon reasonable request.

(1)

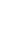




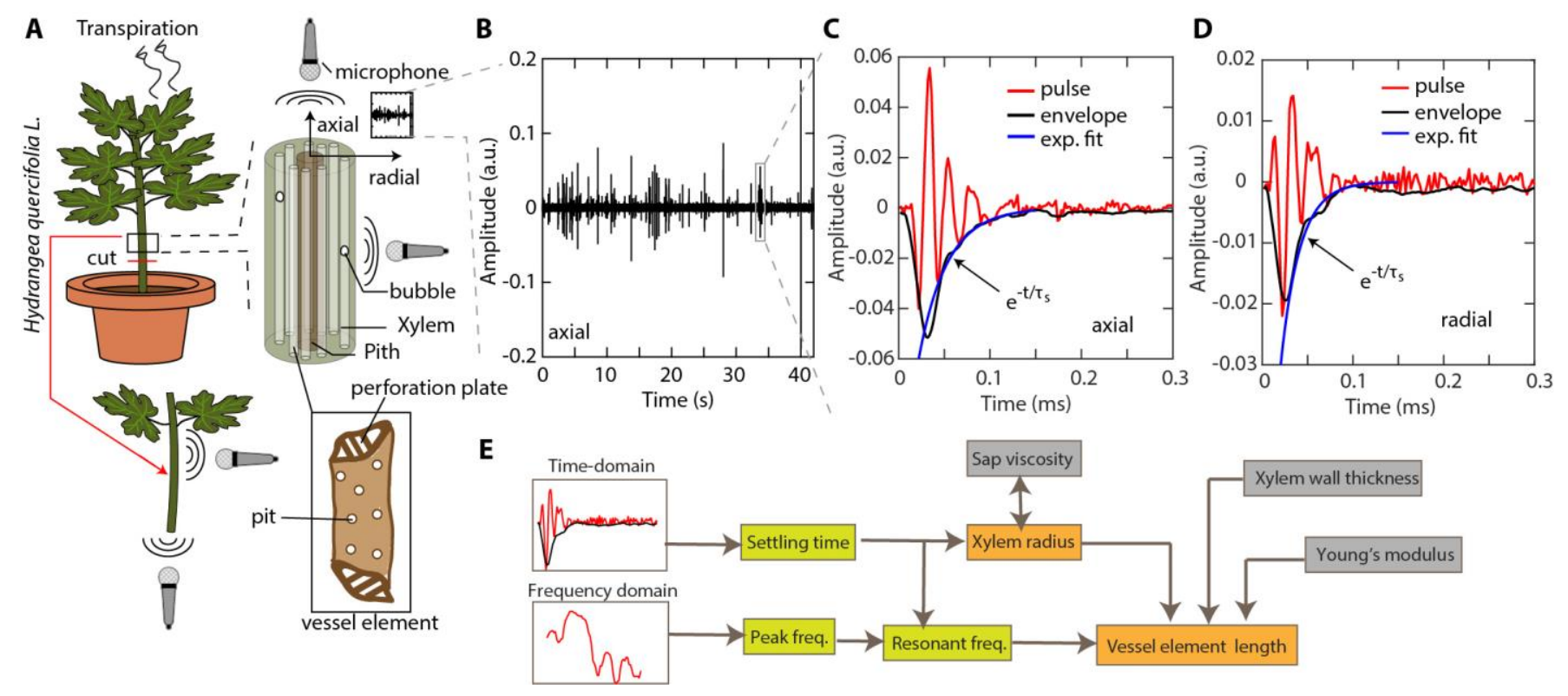

Fig. 1. Ultrasound measurement set-up and time-domain signal. (A) Schematic set-up for recording of ultrasound pulses from shoots of Hydrangea quercifolia along axial and radial directions. The zoom-in represents a schematic of the vascular bundle of the stem, showing the peripheral arrangement of tubular xylem vessels around the pith in the core. Bubbles seeded in the vessels trigger the emission of ultrasound pulses. Each xylem vessel is composed of a network of several vessel elements interconnected via perforation plates as illustrated in the box. (B) Example raw time-domain data for ultrasound recorded along the axial direction of a stem. Time $t=0$ represents the start of the recording, which occurs after 5 minutes of drying. (C), (D) Zoomed-in time-domain example ultrasound pulses recorded axially and radially, respectively. Black curves represent the amplitude envelope that decays exponentially (damping), and blue curves represent the exponential fit of the envelope decay. (E) Schematic flow-chart illustrating the steps in our analysis. The settling time and peak frequencies are obtained from the time-domain and frequency-domain waveforms (Figs. 2E, 2F, 3A, and 3B). The resonant frequency is obtained from peak frequency and settling time (see Materials and Methods). Using these, xylem radius and xylem vessel element length are extracted (Table 1). Parameters of sap viscosity, vessel wall-thickness and young's modulus are taken as input. 

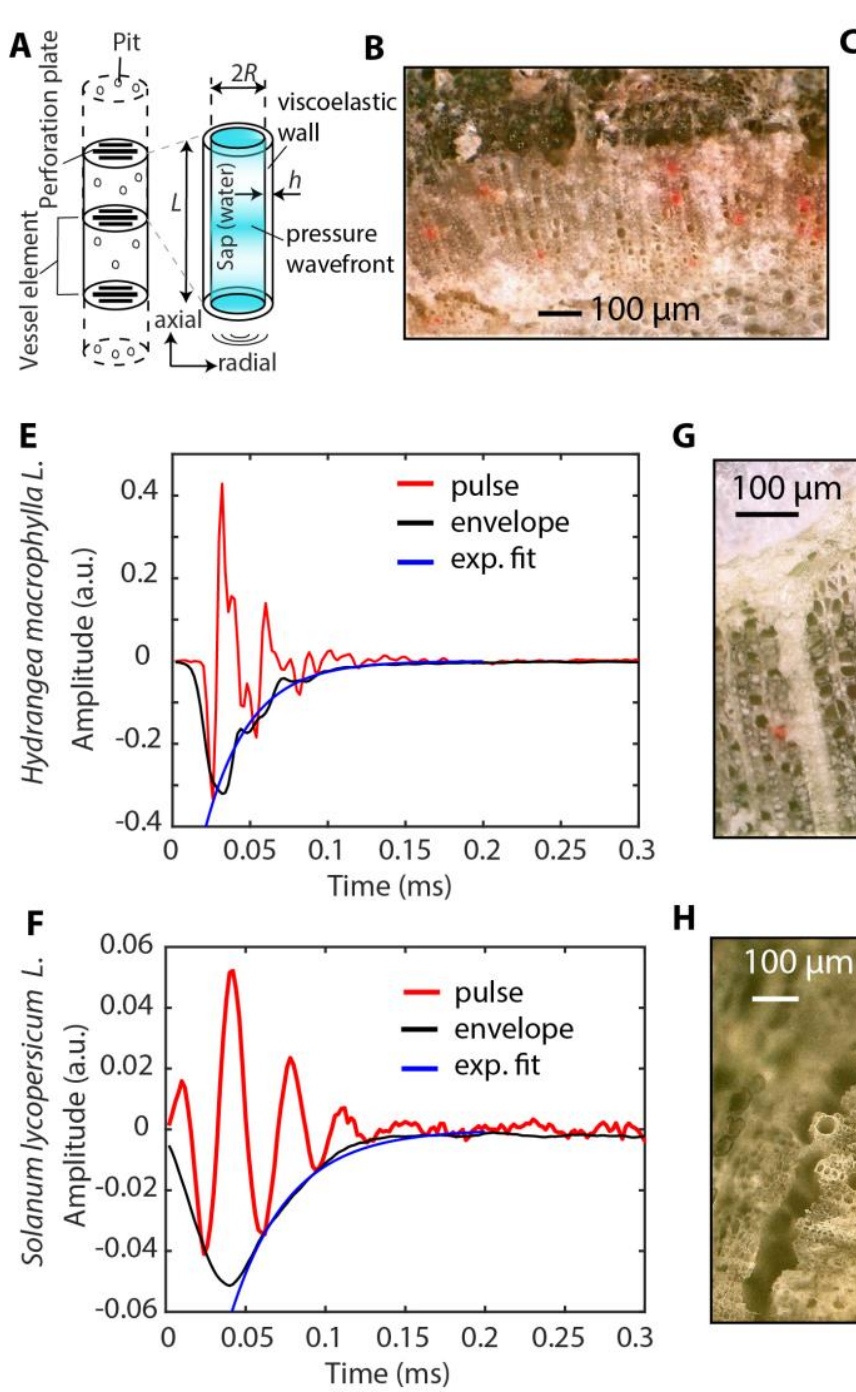
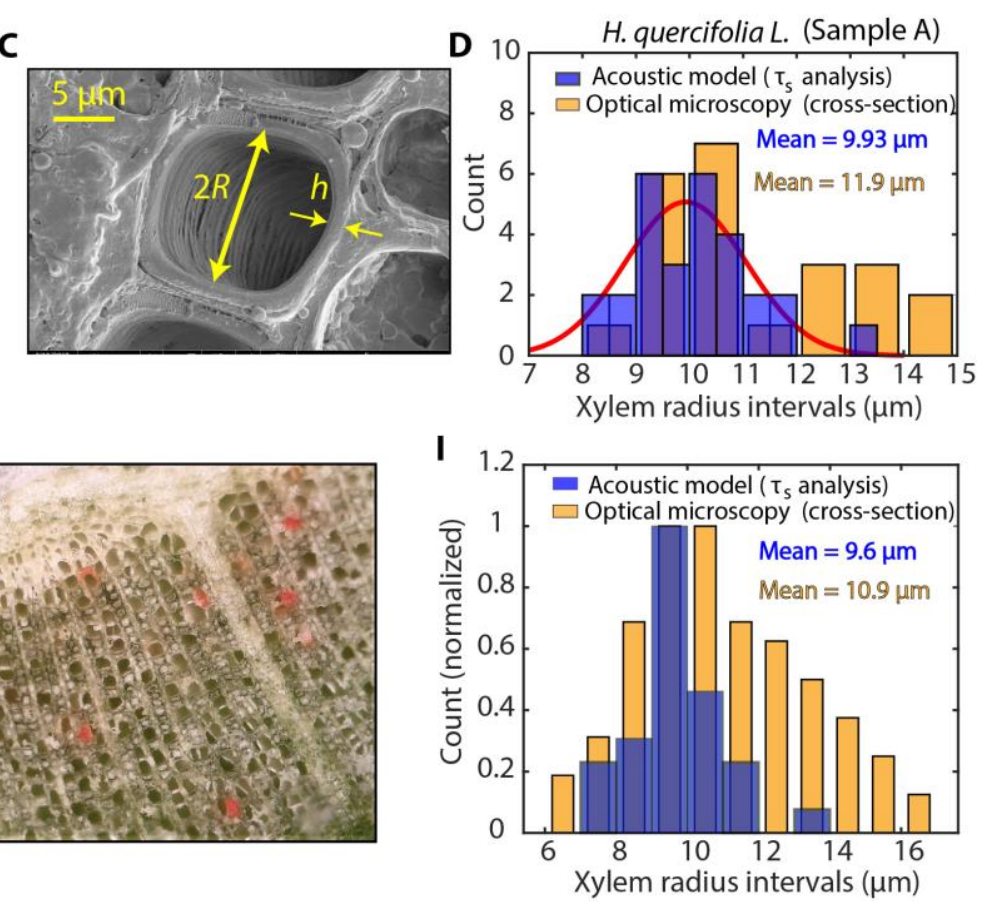

H
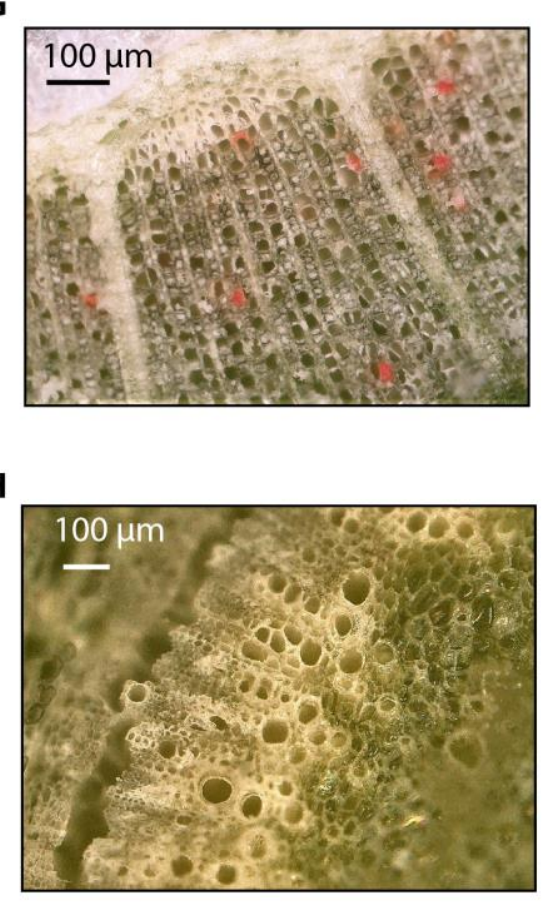

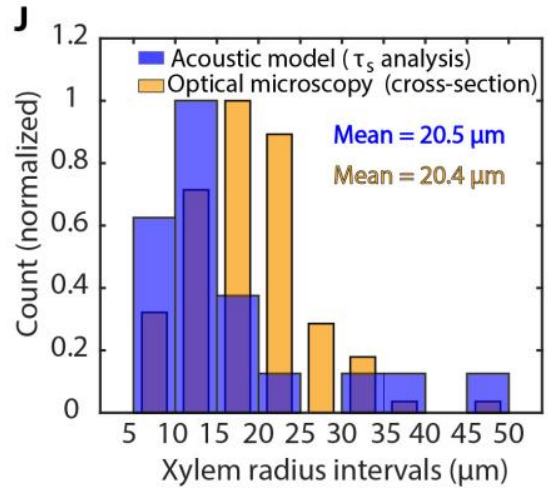

Fig. 2. Xylem vessel radius extraction from damping in axial sound waves. (A) Schematic of water-conducting xylem vessels in vascular plants. Vessels are the dominant and more efficient cells to transport water in H. quercifolia. They consist of a series network of vessel members/elements, which are interconnected via perforation plates. Also shown is the simplified cylindrical acoustic-resonator model for a vessel element sustaining damped longitudinal standing waves in its sap, the damping factor being a function of sap viscosity and the radius of the vessel. (B) Optical micrograph of the transverse section of stem sample A, showing the xylem vessels filled with latex paint. (C) Cryo-SEM image of the transverse section of a stem sample showing the diameter $(2 R)$ and the wall thickness $(h)$ of a xylem vessel. (D) Histogram showing the model-extracted xylem radii (in blue), and that of the observed xylem radii (in yellow) obtained via latex-staining and optical microscopy for stem sample A of H. quercifolia. The red curve represents a unimodal Gaussian fit. (E),(F),(G) Time-domain ultrasound waveform, cross-section optical micrograph (300X) of stem, and histograms of observed (in yellow) and acoustic (in blue) xylem vessel radii, respectively, in H. macrophylla stem sample recorded along the axial direction. (H), (I), (J) Time-domain ultrasound waveform, cross-section optical micrograph (200X) of stem, and histograms of observed (in yellow) and acoustic (in blue) xylem vessel radii, respectively, in Solanum lycopersicum stem sample recorded along the axial direction. 

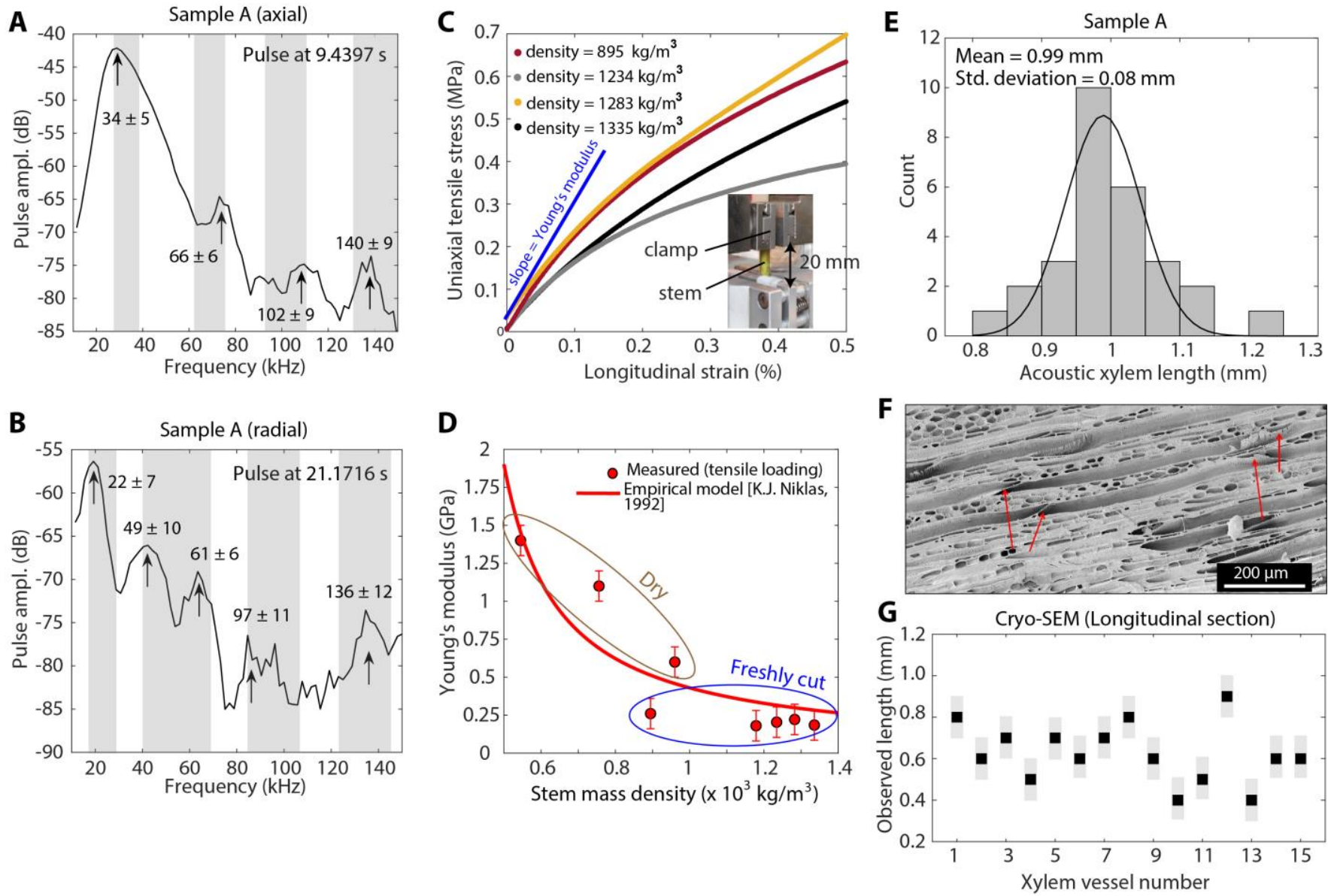

Fig. 3. Ultrasound frequency spectra, Young's moduli of stem, and extraction of xylem vessel element lengths. (A), (B) Observed characteristic peak-frequencies in the example Fourier transform of the ultrasound pulses recorded axially and radially, respectively, in sample A of $H$. quercifolia. The black curve represents the spectrum of a representative pulse with the indicated timestamp of the recording. Grey shaded regions indicate the variation in the peak frequencies among the individual pulses (mean \pm standard deviation). (C) Measured stress-strain curve for freshly cut stem segments of $H$. quercifolia with mass densities indicated in the legend. The blue line represents the linear fit of a stress-strain curve at low strain, the slope of which, yields the Young's modulus in accordance with Hooke's Law. The inset shows the photograph of the set-up for uniaxial tensile loading of the stem. (D) Extracted Young's modulus versus mass density for freshly cut and dried stem segments, extracted from stress-strain measurements (solid circles) with indicated error bars $( \pm 0.1$ $\mathrm{GPa}$ ). The red curve represents a fit based on the empirical model (24) of Young's moduli as a function of relative water-content in stems. (E) Histogram showing the extracted xylem vessel element lengths in stem sample A, extracted via the acoustic model. The black curve represents a unimodal Gaussian fit. (F) Example cryo-SEM image of the longitudinal section of a stem segment (H. quercifolia), showing the structure of individual vessel elements terminated by scalariform perforation plates (marked by red arrows). Observed length of each element is in the range $600 \mu \mathrm{m}-900 \mu \mathrm{m}$. (G) Scatter plot showing the observed length of xylem vessel elements via cryo-SEM technique. The grey bars indicate the upper and lower bounds due to the slant length of the perforation plates along the longitudinal axis $(\sim 100 \mu \mathrm{m})$. 


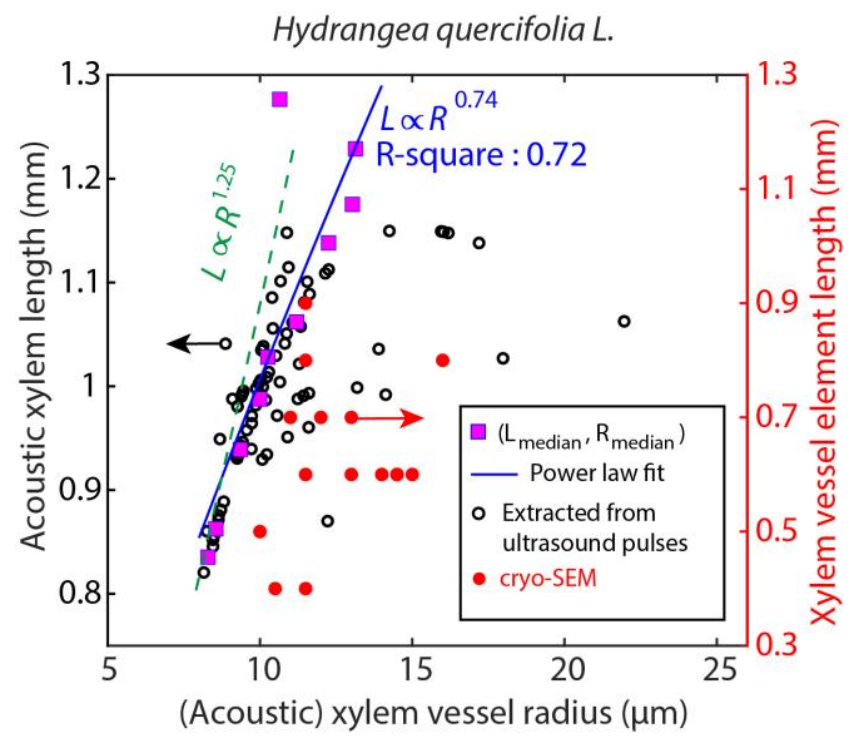

764

765

766

767

768

769

770

771

772

773

774

775

776

777

778

779

780

781

782

783

784

785

786

787

Fig. 4. Acoustic xylem vessel element length versus vessel radius. Black circles: scatter plot of model-extracted (acoustic) xylem vessel element length $(L)$ and radius $(R)$ corresponding to each analysed ultrasound pulse. Data from all three stem samples of $H$. quercifolia are merged here. The radii were obtained from the settling time of the ultrasound pulses, while the lengths were obtained from the resonance frequency of the sound pulses and by measuring the Young's modulus (Fig. 1E). The data points are classified into bins of $L$ with intervals of $0.5 \mathrm{~mm}$. In each bin, the median $L$ and $R$ are calculated (pink squares), and fitted with a power law function (blue line). The green dashed line indicates the predicted $L-R$ dependency in accordance with Darcy-Weisbach equation for fluid flow, combined with mechanical failure of the xylem vessel (see Materials and Methods). Red circles: observed vessel element lengths and radius in $H$. quercifolia via cryo-SEM technique.

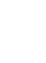


A

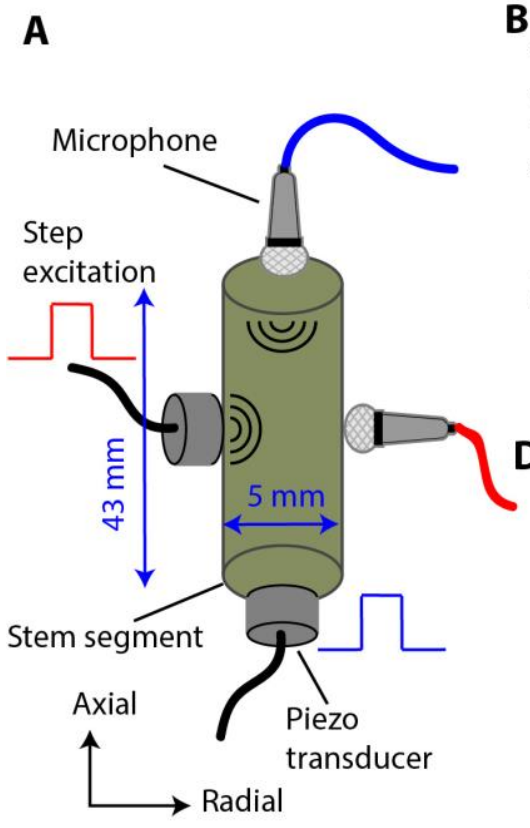

Table 1. Measured versus extracted parameters for $\boldsymbol{H}$. quercifolia. Summary of extracted parameters via the ultrasound analysis, and those obtained by destructive and direct characterization methods, for the three stem samples of $H$. quercifolia.

\begin{tabular}{|l|l|l|l|}
\hline Parameter & $\begin{array}{l}\text { Ultrasound } \\
\text { method }\end{array}$ & \multicolumn{2}{|l|}{ Destructive measurement } \\
\hline Xylem radii $[\mu \mathrm{m}]$ & $9.89 \pm 1.6$ & $12.4 \pm 2.6$ & $\begin{array}{l}\text { Optical microscopy, } \\
\text { Scanning electron } \\
\text { microscopy }\end{array}$ \\
\hline $\begin{array}{l}\text { Mean Xylem vessel } \\
\text { length [mm] }\end{array}$ & --- & $\begin{array}{l}16.9 \text { (sample A), } \\
12.8 \text { (sample B), } \\
14.4 \text { (sample C). }\end{array}$ & $\begin{array}{l}\text { Latex-paint staining and } \\
\text { vessel counting }\end{array}$ \\
\hline $\begin{array}{l}\text { Xylem vessel element } \\
\text { length [mm] }\end{array}$ & $0.98 \pm 0.14$ & $0.63 \pm 0.14$ & $\begin{array}{l}\text { Scanning electron } \\
\text { microscopy }\end{array}$ \\
\hline $\begin{array}{l}\text { Young's modulus of } \\
\text { elasticity [GPa] }\end{array}$ & --- & $0.2 \pm 0.1$ & Uniaxial tensile loading \\
\hline
\end{tabular}
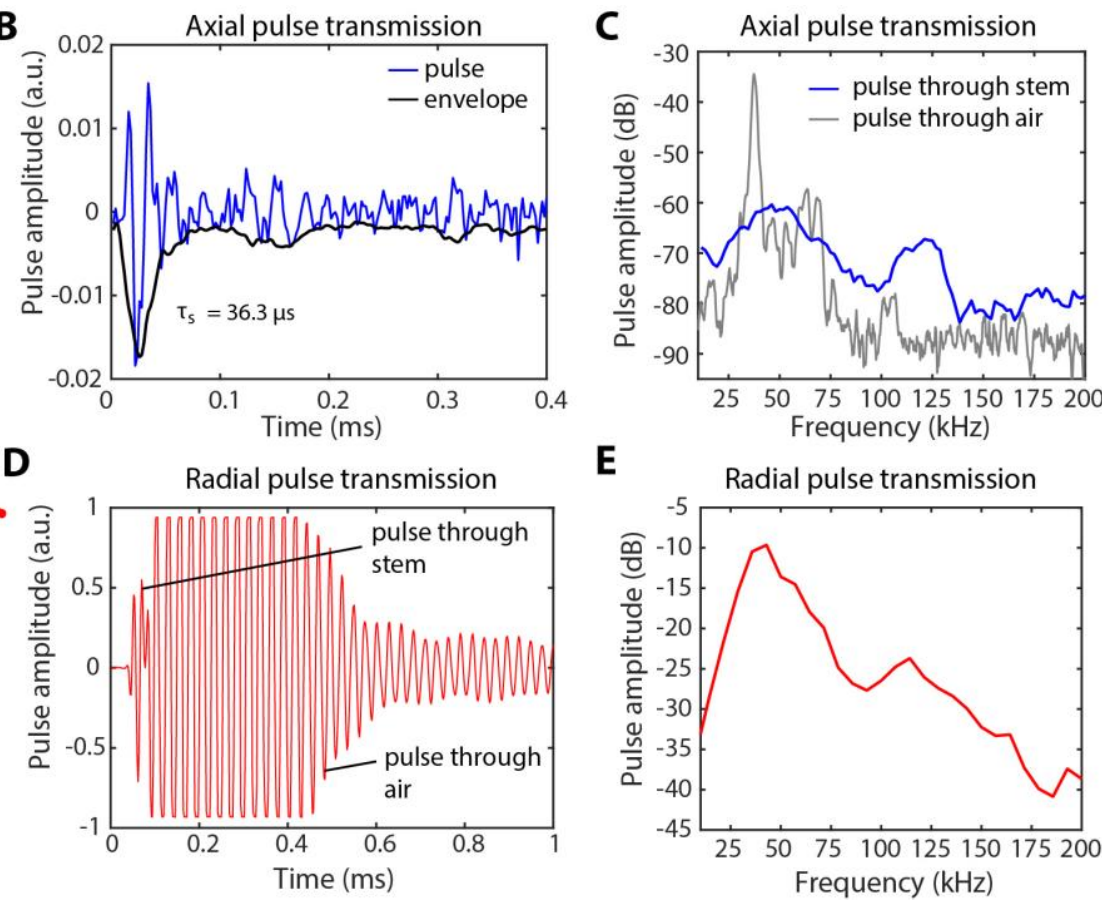

Fig. 5. Ultrasound pulsed transmission spectroscopy of vascular tissue. (A) Schematic measurement set-up showing an external piezo-ultrasound transducer (resonant frequency of $40 \mathrm{kHz}$ ) and the broad-band microphone placed along the axial and radial direction to a $H$. macrophylla stem with the indicated dimensions. The piezo-transducer is excited with a voltage step in order to emit a broad-band acoustic pulse. (B) Time-domain waveform (in 
blue) with the fitted envelope, and (C) Fourier spectra of the axially transmitted sound pulse (in blue). The spectrum of the sound pulse emitted by the transducer (source) with

801 transmission through air is shown in grey as a reference. (D) Time-domain waveform and (E) 802 Fourier spectra of the radially transmitted sound pulse.

803 


\section{Figures}
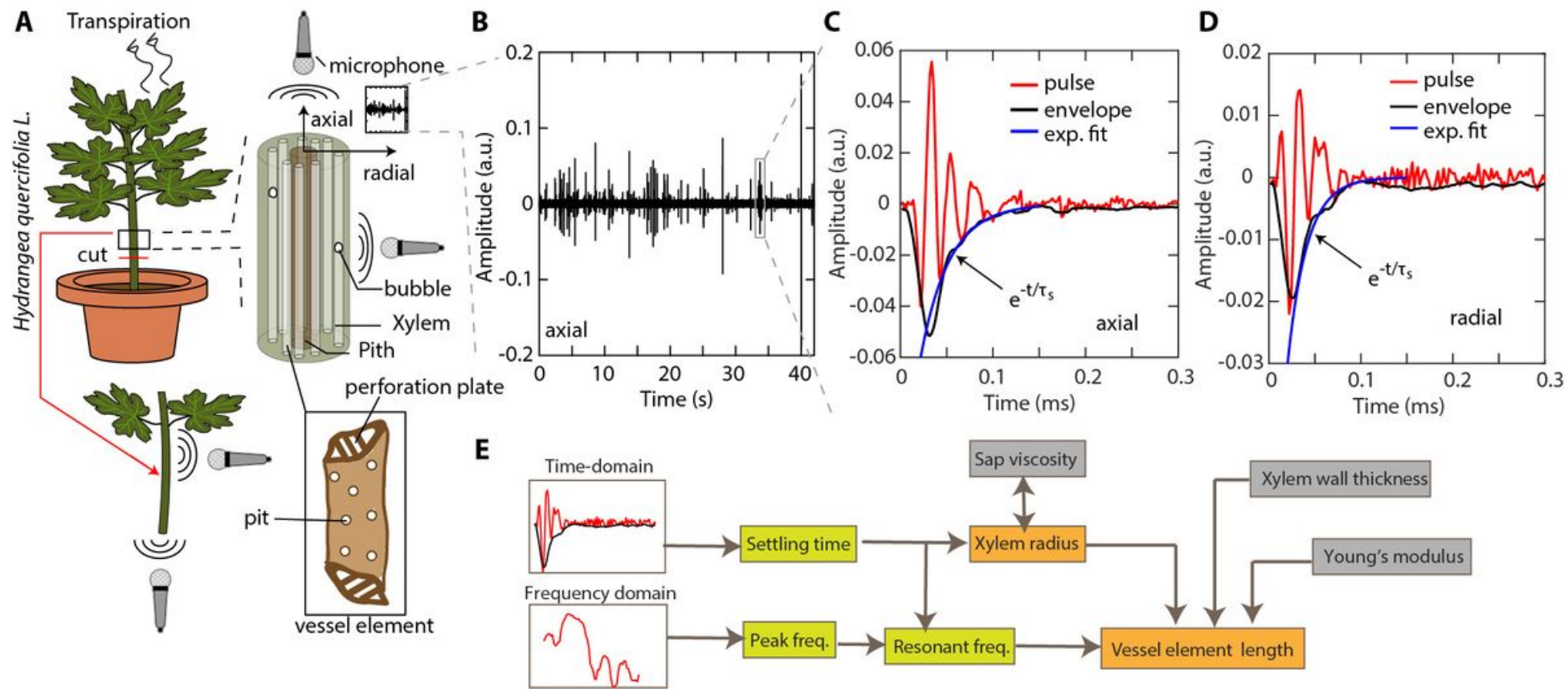

\section{Figure 1}

Ultrasound measurement set-up and time-domain signal. (A) Schematic set-up for recording of ultrasound pulses from shoots of Hydrangea quercifolia along axial and radial directions. The zoom-in represents a schematic of the vascular bundle of the stem, showing the peripheral arrangement of tubular xylem vessels around the pith in the core. Bubbles seeded in the vessels trigger the emission of ultrasound pulses. Each xylem vessel is composed of a network of several vessel elements interconnected via perforation plates as illustrated in the box. (B) Example raw time-domain data for ultrasound recorded along the axial direction of a stem. Time $t=0$ represents the start of the recording, which occurs after $\sim 5$ minutes of drying. (C), (D) Zoomed-in time-domain example ultrasound pulses recorded axially and radially, respectively. Black curves represent the amplitude envelope that decays exponentially (damping), and blue curves represent the exponential fit of the envelope decay. $(E)$ Schematic flow-chart illustrating the steps in our analysis. The settling time and peak frequencies are obtained from the time-domain and frequency-domain waveforms (Figs. 2E, 2F, 3A, and 3B). The resonant frequency is obtained from peak frequency and settling time (see Materials and Methods). Using these, xylem radius and xylem vessel element length are extracted (Table 1). Parameters of sap viscosity, vessel wall-thickness and young's modulus are taken as input. 


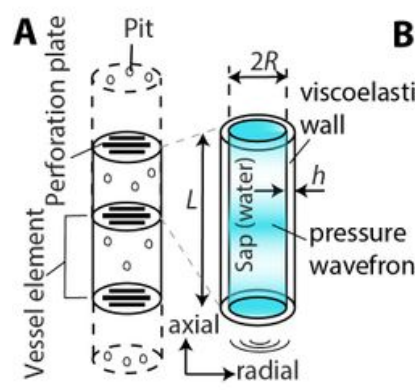

B
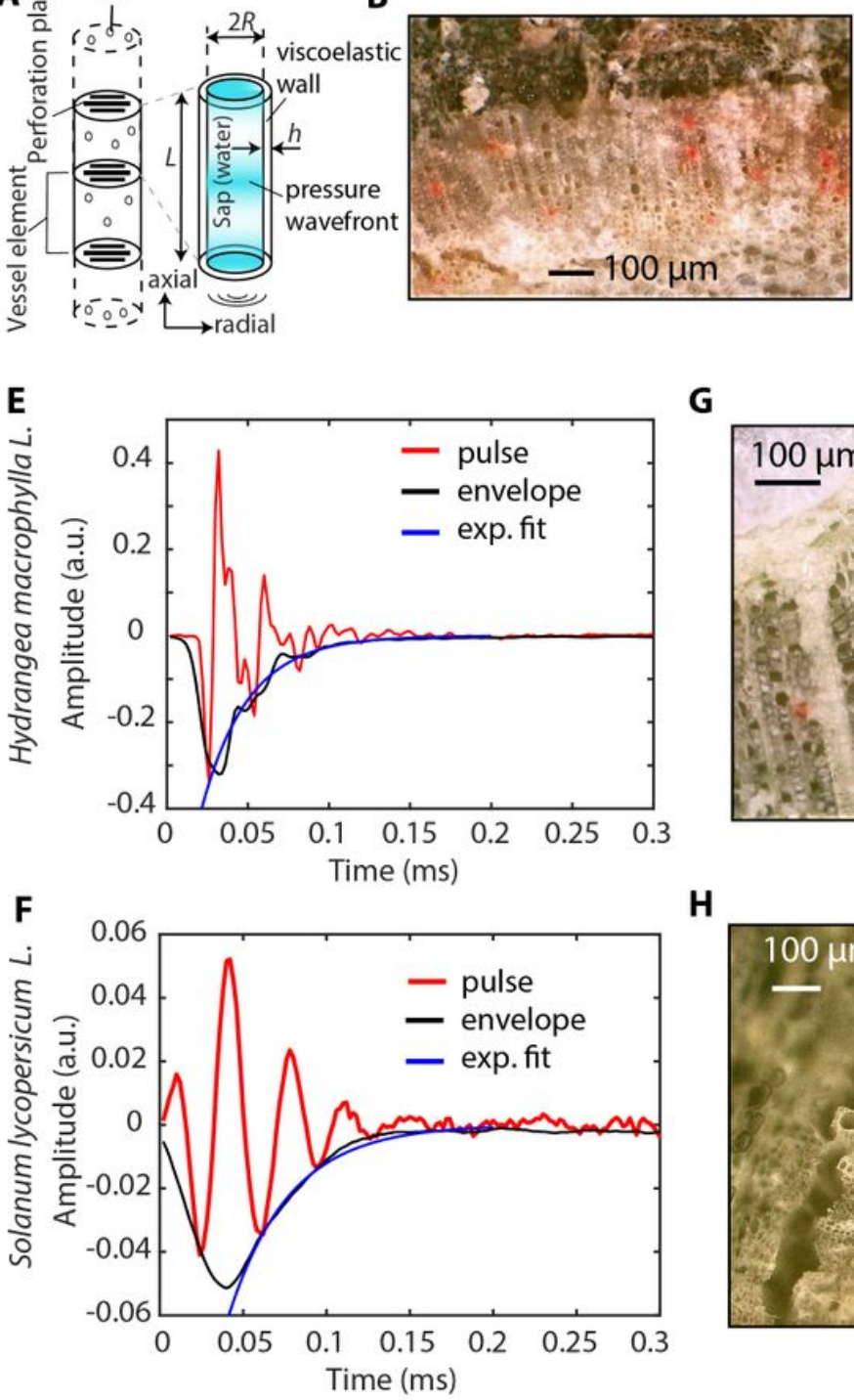

C

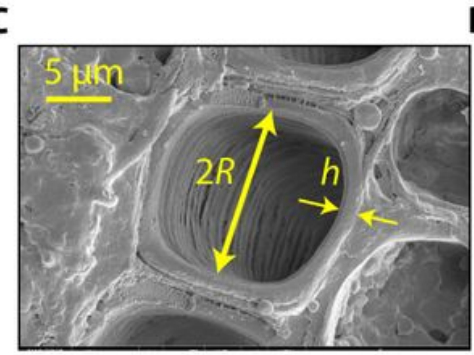

G
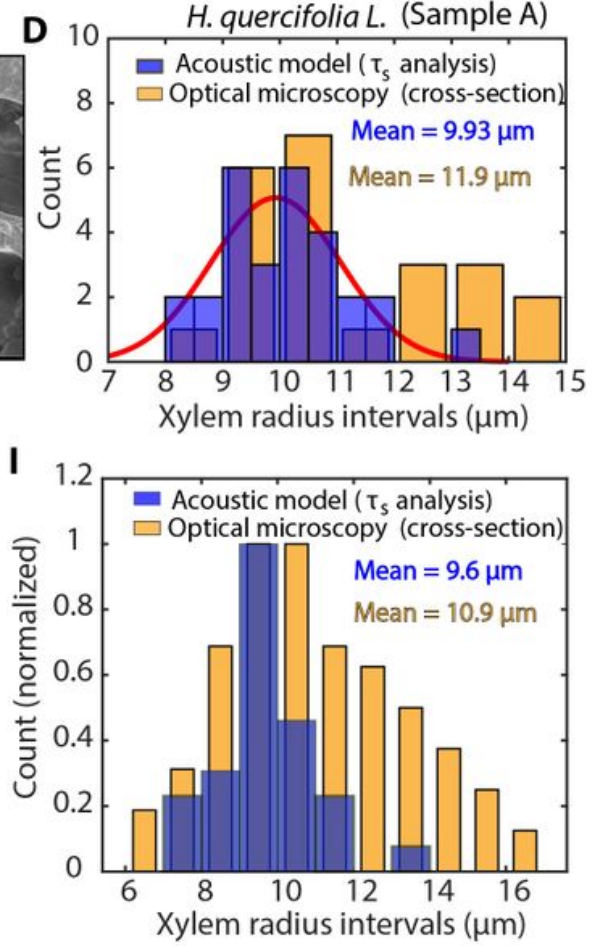

H
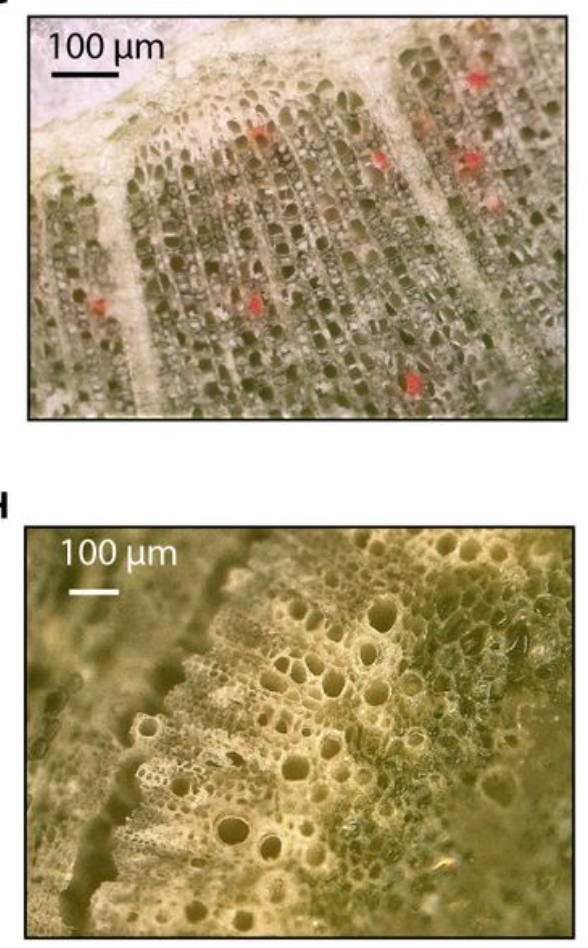

J

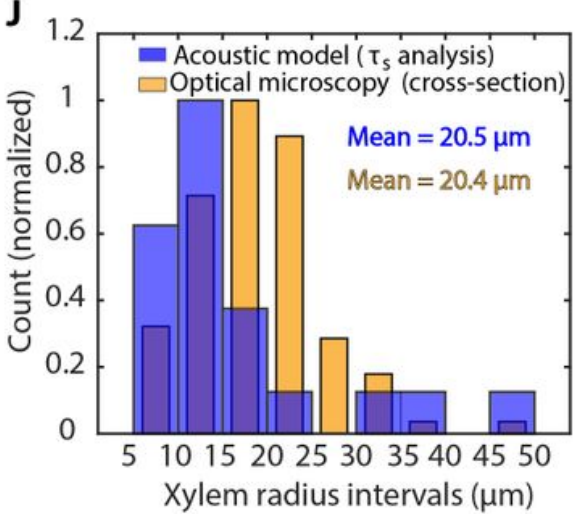

Figure 2

Xylem vessel radius extraction from damping in axial sound waves. (A) Schematic of water-conducting xylem vessels in vascular plants. Vessels are the dominant and more efficient cells to transport water in $\mathrm{H}$. quercifolia. They consist of a series network of vessel members/elements, which are interconnected via perforation plates. Also shown is the simplified cylindrical acoustic-resonator model for a vessel element sustaining damped longitudinal standing waves in its sap, the damping factor being a function of sap viscosity and the radius of the vessel. (B) Optical micrograph of the transverse section of stem sample A, showing the xylem vessels filled with latex paint. (C) Cryo-SEM image of the transverse section of a stem sample showing the diameter (2R) and the wall thickness (h) of a xylem vessel. (D) Histogram showing the model-extracted xylem radii (in blue), and that of the observed xylem radii (in yellow) obtained via latex-staining and optical microscopy for stem sample A of H. quercifolia. The red curve represents a unimodal Gaussian fit. (E),(F),(G) Time-domain ultrasound waveform, cross-section optical micrograph (300X) of stem, and histograms of observed (in yellow) and acoustic (in blue) xylem vessel radii, respectively, in $\mathrm{H}$. macrophylla stem sample recorded along the axial direction. $(\mathrm{H}),(\mathrm{I}),(\mathrm{J})$ Time- 
domain ultrasound waveform, cross-section optical micrograph (200X) of stem, and histograms of observed (in yellow) and acoustic (in blue) xylem vessel radii, respectively, in Solanum lycopersicum stem sample recorded along the axial direction.
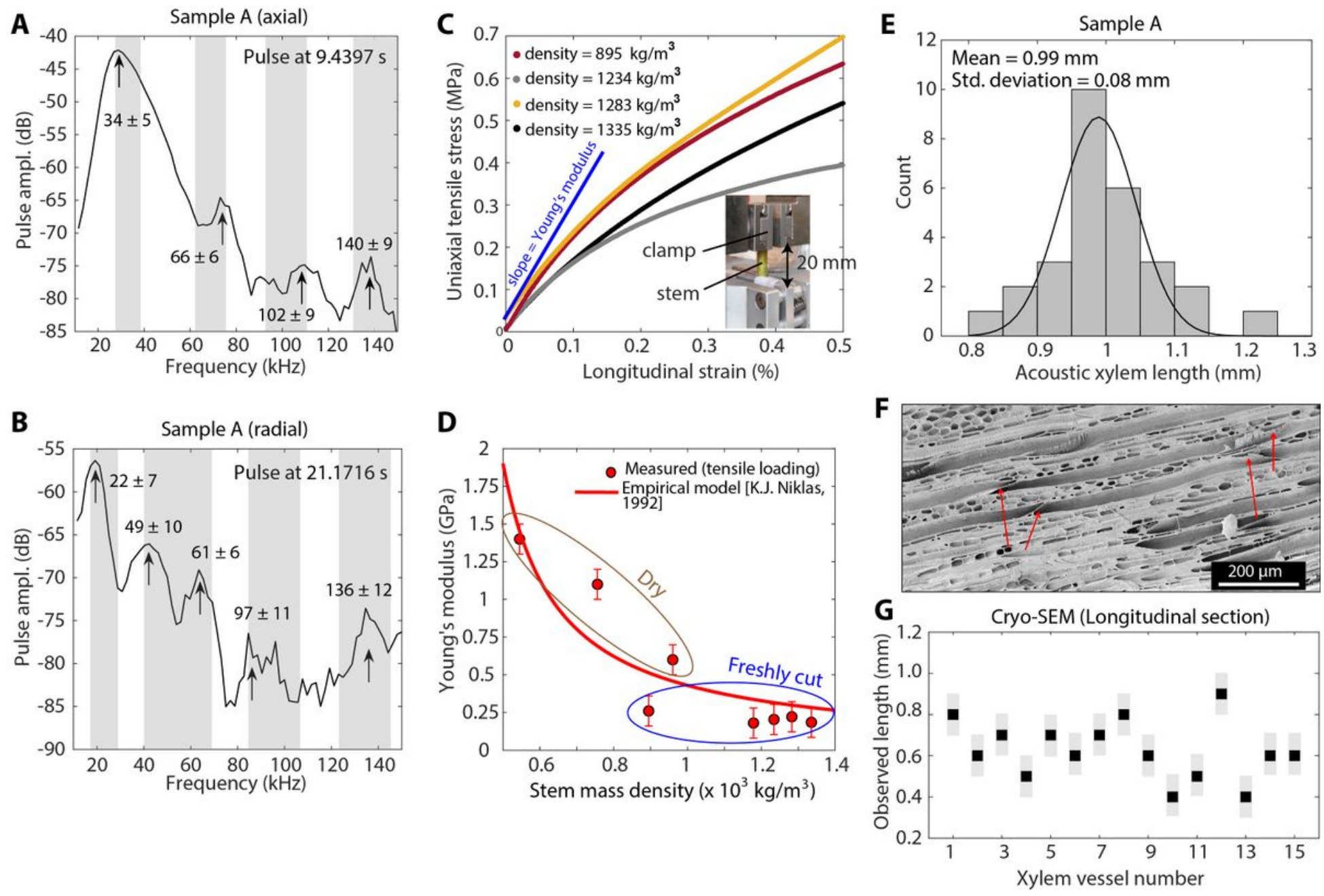

\section{Figure 3}

Ultrasound frequency spectra, Young's moduli of stem, and extraction of xylem vessel element lengths. (A), (B) Observed characteristic peak-frequencies in the example Fourier transform of the ultrasound pulses recorded axially and radially, respectively, in sample $\mathrm{A}$ of $\mathrm{H}$. quercifolia. The black curve represents the spectrum of a representative pulse with the indicated timestamp of the recording. Grey shaded regions indicate the variation in the peak frequencies among the individual pulses (mean \pm standard deviation). (C) Measured stress-strain curve for freshly cut stem segments of $\mathrm{H}$. quercifolia with mass densities indicated in the legend. The blue line represents the linear fit of a stress-strain curve at low strain, the slope of which, yields the Young's modulus in accordance with Hooke's Law. The inset shows the photograph of the set-up for uniaxial tensile loading of the stem. (D) Extracted Young's modulus versus mass density for freshly cut and dried stem segments, extracted from stress-strain measurements (solid circles) with indicated error bars $( \pm 0.1 \mathrm{GPa})$. The red curve represents a fit based on the empirical model (24) of Young's moduli as a function of relative water-content in stems. (E) Histogram showing the extracted xylem vessel element lengths in stem sample A, extracted via the acoustic model. The black 
curve represents a unimodal Gaussian fit. (F) Example cryo-SEM image of the longitudinal section of a stem segment $(\mathrm{H}$. quercifolia), showing the structure of individual vessel elements terminated by scalariform perforation plates (marked by red arrows). Observed length of each element is in the range $600 \mu \mathrm{m}-900 \mu \mathrm{m}$. (G) Scatter plot showing the observed length of xylem vessel elements via cryo-SEM technique. The grey bars indicate the upper and lower bounds due to the slant length of the perforation plates along the longitudinal axis $(\sim 100 \mu \mathrm{m})$.

\section{Hydrangea quercifolia $L$.}

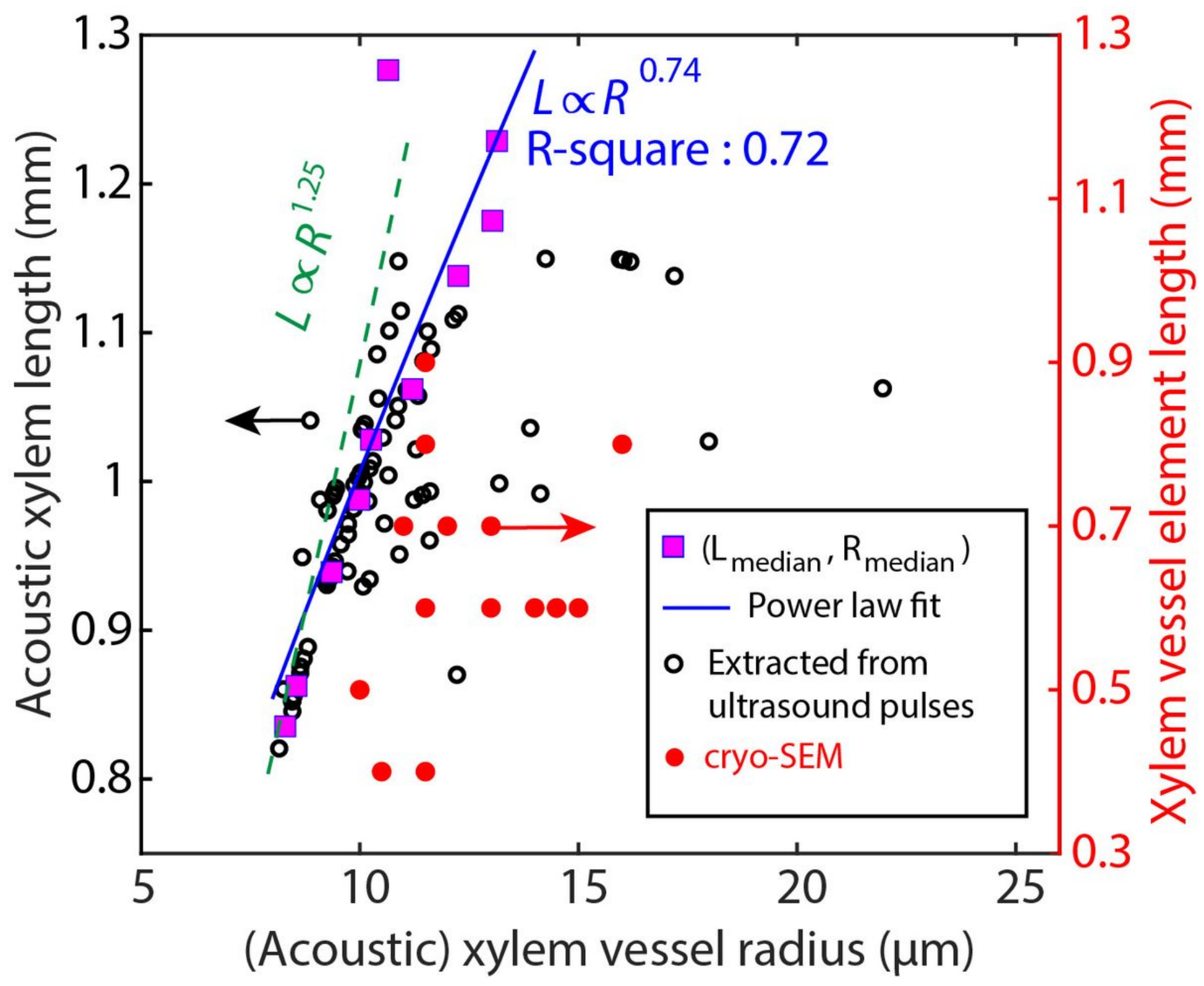

Figure 4

Acoustic xylem vessel element length versus vessel radius. Black circles: scatter plot of model-extracted (acoustic) xylem vessel element length $(L)$ and radius $(R)$ corresponding to each analysed ultrasound pulse. Data from all three stem samples of $\mathrm{H}$. quercifolia are merged here. The radii were obtained from the settling time of the ultrasound pulses, while the lengths were obtained from the resonance frequency 
of the sound pulses and by measuring the Young's modulus (Fig. 1E). The data points are classified into bins of $L$ with intervals of $0.5 \mathrm{~mm}$. In each bin, the median $L$ and $R$ are calculated (pink squares), and fitted with a power law function (blue line). The green dashed line indicates the predicted L-R dependency in accordance with Darcy-Weisbach equation for fluid flow, combined with mechanical failure of the xylem vessel (see Materials and Methods). Red circles: observed vessel element lengths and radius in $\mathrm{H}$. quercifolia via cryo-SEM technique.
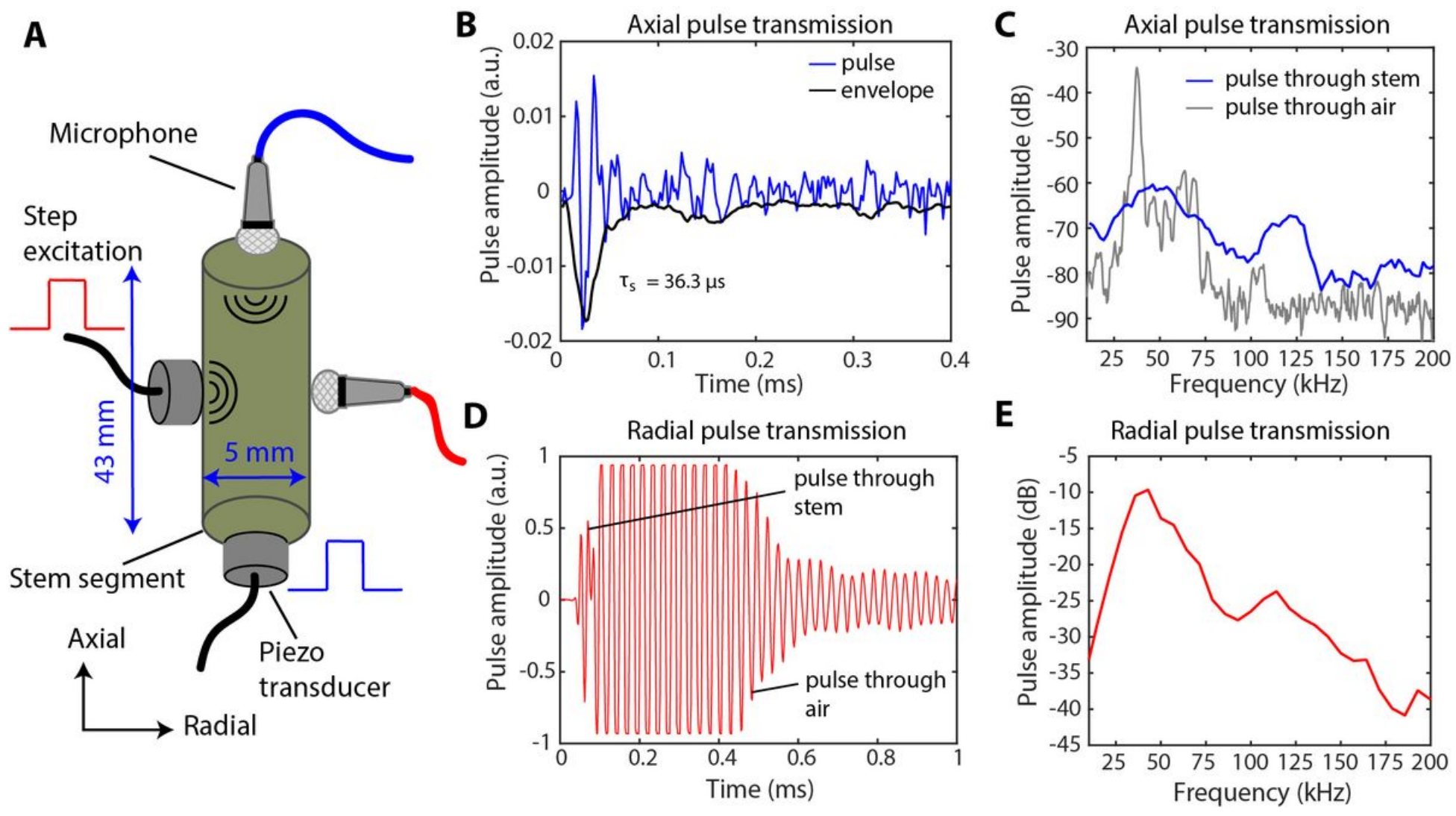

Figure 5

Ultrasound pulsed transmission spectroscopy of vascular tissue. (A) Schematic measurement set-up showing an external piezo-ultrasound transducer (resonant frequency of $40 \mathrm{kHz}$ ) and the broad-band microphone placed along the axial and radial direction to a $\mathrm{H}$. macrophylla stem with the indicated dimensions. The piezo-transducer is excited with a voltage step in order to emit a broad-band acoustic pulse. (B) Time-domain waveform (in blue) with the fitted envelope, and (C) Fourier spectra of the axially transmitted sound pulse (in blue). The spectrum of the sound pulse emitted by the transducer (source) with transmission through air is shown in grey as a reference. (D) Time-domain waveform and (E) Fourier spectra of the radially transmitted sound pulse.

\section{Supplementary Files}

This is a list of supplementary files associated with this preprint. Click to download.

- Supplementarylnfo.docx 